The Astronomical Journal, 121:3026-3040, 2001 June

(C) 2001. The American Astronomical Society. All rights reserved. Printed in U.S.A.

\title{
THE NEUTRAL INTERSTELLAR MEDIUM OF THE DWARF IRREGULAR GALAXY DDO 47 AND ITS COMPANION
}

\author{
FABIAN WALTER ${ }^{1}$ \\ Department of Astronomy, 105-24, California Institute of Technology, 1201 East California Boulevard, Pasadena, CA 91125; fw@astro.caltech.edu \\ AND \\ ELIAS BRINKS \\ Departamento de Astronomía, Apartado Postal 144, Guanajuato, GTO 36000, Mexico; ebrinks@astro.ugto.mx \\ Received 2000 May 11; accepted 2001 February 14
}

\begin{abstract}
We present high-resolution $\mathrm{H}_{\mathrm{I}}$ observations obtained with the Very Large Array, as well as optical broadband imaging, of the dwarf irregular galaxy DDO 47 (UGC 3974, CGCG 087-030). The H I maps show a variety of structures, such as holes and shells, including one supergiant shell, in its neutral interstellar medium. We derive an $\mathrm{H}$ I mass for DDO 47 of $2.3 \times 10^{8} M_{\odot}$. We cataloged $19 \mathrm{H}$ I holes in DDO 47, about half of them showing signs of expansion. According to the "standard model," their origin is believed to be due to past regions of massive star formation through the effects of stellar winds and supernova explosions. A comparison of $\mathrm{H}$ II regions with the positions of the $\mathrm{H}$ I holes yields that most of the current star-forming regions are situated near the rims of the larger $\mathrm{H}$ I holes, indicative of propagating star formation. The mean properties of the $\mathrm{H}$ I holes in DDO 47 are very much like those found in other dwarf galaxies. At a projected distance of $20 \mathrm{kpc}$ (adopting a distance to DDO 47 of $4 \mathrm{Mpc}$ ), a galaxy that is cataloged as CGCG 087-033 was found to be at almost the same systemic velocity as DDO 47 . This object is a likely companion, with an $\mathrm{H}$ I mass of $1.4 \times 10^{7} M_{\odot}$ and a dynamical mass of at least $1.2 \times 10^{8} M_{\odot}$. A dynamical study of DDO 47 reveals that this object requires dark matter at a level of about $80 \%$ of its dynamical mass $\left(M_{\mathrm{dyn}} \approx 5 \times 10^{9} M_{\odot}\right)$ to explain the observed rotation (assuming a stellar mass-to-light ratio of $1 M_{\odot} / L_{B \odot}$ ). Using a simple mass model, the scale height of the $\mathrm{H}$ I distribution of DDO 47 is derived to be about $500 \mathrm{pc}$. This high value is similar to values found in other dwarf irregular galaxies and can be explained as being due to a lower gravitational potential. In summary, DDO 47 is a gas-rich dwarf irregular galaxy that shows a rather complex structure in its neutral interstellar medium. We speculate that the flyby of the companion may have triggered a starburst in DDO 47 in the past, which may have created the $\mathrm{H}$ I holes that are now present.

Key words: galaxies: individual (DDO 47, CGCG 087-033) — galaxies: irregular - galaxies: ISM ISM: bubbles - ISM: H I - ISM: structure - radio emission lines
\end{abstract}

\section{INTRODUCTION}

In recent years, people have been turning increasingly to the most numerous type of galaxy, dwarf galaxies, to study such important questions as how galaxies form, evolve, and interact with one another, to determine which are the necessary and sufficient conditions for star formation to commence, and to shed light on the nature of dark matter. In addition, given that in today's most popular models for galaxy formation ("bottom-up scenarios") gas-rich, lowmetallicity dwarf galaxies play a key role, detailed studies of nearby specimens are invaluable, since they provide a unique opportunity to study objects in our local universe that may have dominated events at larger look-back times. In this paper, we will discuss what can be learned from a study of the distribution and kinematics of the neutral atomic hydrogen in one of these systems and concentrate on the structure of the interstellar medium (ISM) as mapped at a resolution of the order of $100 \mathrm{pc}$.

We know from observations of spiral galaxies that regions of star formation (SF), especially of massive stars (i.e., those whose masses are larger than $8 M_{\odot}$ ), which after

\footnotetext{
${ }^{1}$ Visiting Astronomer, German-Spanish Astronomical Centre, Calar Alto, operated by the Max-Planck-Institut für Astronomie (Heidelberg) jointly with the Spanish Comisión Nacional de Astronomía.
}

a relatively short life go off as supernovae, have a profound influence on the surrounding ISM. In the "standard" picture, these massive stars are thought to produce largescale structures in the form of expanding "holes" and "shells," which are evident in maps of neutral and ionized hydrogen. These holes and shells delineate cavities that are supposedly filled with coronal gas, created by a central stellar cluster that drives the expansion of the shell. New star formation can eventually start on the rims of these expanding structures once densities exceed a certain threshold. Hence, the late stages of the evolution of the most massive stars provide an important link in the feedback loop that triggers and regulates star formation (for review articles on this topic, see Tenorio-Tagle \& Bodenheimer 1988; van der Hulst 1996; Brinks \& Walter 1998). This is very much as envisaged in earlier models of stochastic selfpropagating star formation (SSPSF; for early papers on this topic, see Mueller \& Arnett 1976; Gerola \& Seiden 1978). In dwarf galaxies, where spiral density waves do not play a role, SSPSF might be the only process that effectively keeps star formation going.

Several groups are working on numerical simulations and theoretical models to put to the test some models of SSPSF as applied to Magellanic irregular galaxies (see, e.g., Palouš 1998, and references therein). Having said that, it should be noted that the standard model for the creation of (super)giant shells through stellar winds and supernova 
(SN) explosions of the most massive stars in OB associations is currently being questioned by some authors (e.g., Rhode, Salzer, \& Westpfahl 1999).

A key ingredient in the above mentioned studies are observations in the $21 \mathrm{~cm}$ line of neutral hydrogen $(\mathrm{H} \mathrm{I})$. However, it is only recently that enough high-resolution $\mathrm{H} \mathrm{I}$ studies of galaxies have become available to allow a meaningful comparison to be made of the structures in the ISM, e.g., as a function of Hubble type. Prominent examples are M31 (Brinks 1981; Brinks \& Bajaja 1986), M33 (Deul \& den Hartog 1990), Holmberg II (Ho II; Puche et al. 1992), the galaxies M101 and NGC 6946 (Kamphuis 1993), IC 10 (Wilcots \& Miller 1998), IC 2574 (Walter \& Brinks 1999), Holmberg I (Ho I; Ott et al. 2000), and NGC 6822 (de Blok \& Walter 2000). Impressive additions to this list at even higher spatial resolution are the Magellanic Clouds (SMC: Staveley-Smith et al. 1997; LMC: Kim et al. 1998).

Gas-rich dwarf galaxies (dwarf irregulars) are also ideal laboratories to study dark matter (DM) by means of $\mathrm{H} \mathrm{I}$ observations. This is because these galaxies are dynamically much simpler systems as compared with high-mass spiral galaxies. They lack a bulge component, and their $\mathrm{H}$ I mass is often comparable to that in stars. This reduces the number of free parameters resulting in a more reliable determination of the dark matter content. A potential disadvantage is that dwarf irregular galaxies are dominated by solid-body rotation, which hampers the determination of their inclination. The picture that has emerged in recent years is that gas-rich dwarf galaxies, in general, have a significant dark matter content, and this DM dominates the dynamics of the galaxy throughout (see, e.g., the studies by McGaugh \& de Blok 1997, Swaters et al. 1998, and Stil 1999, to name but a few). This is in contrast to spiral galaxies, in which the DM only becomes an important fraction of the total mass at larger radii.

In addition, much can be learned from bursts of SF in dwarf galaxies: the intensity of the violent star formation episodes, as seen in a subclass of dwarf galaxies, the socalled blue compact dwarf galaxies (BCD or $\mathrm{H}$ II galaxies), implies that they can only be transient events. One of the ways in which star formation can be triggered in a galaxy is by gravitational interaction with a close neighbor-or by the less obvious harassment by a distant one (see, e.g., Walter et al. 1997). Taylor (1997) argues that dwarf galaxies that show a starburst have a higher probability of having a companion than those who currently do not experience star formation, such as low surface brightness dwarf galaxies. However, these results still suffer from low statistics.

The purpose of the observations presented here is to add another dwarf irregular galaxy to the sample now available in the literature in order to contribute to our understanding of the structure of the ISM in (dwarf) galaxies and its dependence on Hubble type, the importance of DM in dwarfs, and the role that is played by companions in, for example, triggering star formation through interactions. We chose DDO 47, a nearby galaxy that to date has not received the attention it deserves, and report on high-resolution $\mathrm{H} \mathrm{I}_{\mathrm{I}}$ observations of its ISM.

\section{THE DWARF GALAXY DDO 47}

\subsection{Previous Studies}

To date, DDO 47 (also known as UGC 3974) has not been featured as the subject of a paper. However, numerous authors have included it in their samples, such as Hoffman et al. (1996), who collected H I observations of a large sample of dwarf irregular galaxies using the Arecibo singledish telescope. Their sample included DDO 47, which turned out to be one of the most extended dwarf galaxies studied thus far. Despite the low resolution of Arecibo (about $3^{\prime}$ ), they were able to create maps and derive a rotation curve (this will be discussed in $\S 5$ ). Other single-dish $\mathrm{H}$ I measurements of DDO 47 are included in the catalogs by Huchtmeier \& Richter (1988), Lewis, Helou, \& Salpeter (1985), and Hunter \& Gallagher (1985a). CO and heavy element abundance studies of DDO 47 have been published, e.g., by Young et al. (1995), Skillman, Kennicutt, \& Hodge (1989), and Tacconi \& Young (1987). Regarding $\mathrm{CO}$, only a weak detection was reported by Young et al. (1995). Surveys of extragalactic H II regions that include DDO 47 were published by Youngblood \& Hunter (1999), Hunter, Hawley, \& Gallagher (1993), Strobel, Hodge, \& Kennicutt (1991), and Hunter \& Gallagher (1985b). Some general properties of DDO 47 are summarized in Table 1.

\subsection{Distance to DDO 47}

Unfortunately, estimates for the distance to DDO 47 span a rather wide range in the literature from 2.1 to 5.6 Mpc (see, e.g., de Vaucouleurs, de Vaucouleurs, \& Buta 1983; Skillman et al. 1989). The low value adopted by de Vaucouleurs et al. was indirectly obtained based on apparent group membership of galaxies. Assuming a systemic velocity of $155 \mathrm{~km} \mathrm{~s}^{-1}$ for DDO 47, they situated the object in the NGC 2683 group of galaxies and assigned it a distance of 2.1 Mpc. However, since their systemic velocity of DDO 47 is too low by about $130 \mathrm{~km} \mathrm{~s}^{-1}$ (see $\S 4.1$ ), their adopted distance is incorrect and the real distance is probably larger. Georgiev, Karachentsev, \& Tikhonov (1997) determined a distance of $4.26 \mathrm{Mpc}$ to DDO 47. However, this determination was based on one star only (I. Karachentsev 1998, private communication). Several other authors have estimated the distance toward DDO 47, assuming it to be a field galaxy and assuming Hubble flow, using various values for the Hubble constant $H_{0}$. For a systemic velocity $v_{\text {sys }}=280 \mathrm{~km} \mathrm{~s}^{-1}$, these estimates range

TABLE 1

GENERAL INFORMATION ON DDO 47

\begin{tabular}{|c|c|}
\hline Parameters & Values \\
\hline Object ........ & DDO 47 \\
\hline Other names...... & UGC 3974, CGCG $087-030$ \\
\hline 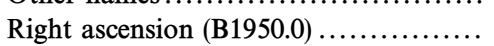 & $07^{\mathrm{h}} 39^{\mathrm{m}} 03^{\mathrm{s}} .0$ \\
\hline Declination $(\mathrm{B} 1950.0) \ldots \ldots \ldots \ldots \ldots \ldots \ldots$ & $16^{\circ} 55^{\prime} 07^{\prime \prime}$ \\
\hline Adopted distance $^{\mathrm{a}} \ldots \ldots \ldots \ldots \ldots \ldots \ldots$ & $4 \mathrm{Mpc}$ \\
\hline Inclination $^{\mathrm{b}} \ldots \ldots \ldots \ldots \ldots \ldots \ldots \ldots$ & $35^{\circ} \pm 3^{\circ}$ \\
\hline Scale $\left(1^{\prime \prime}\right.$ corresponds to $) \ldots \ldots \ldots \ldots \ldots$ & $19.4 \mathrm{pc}$ \\
\hline Corrected apparent magnitude $(B)^{\mathfrak{c}} \ldots \ldots$ & $13.5 \mathrm{mag}$ \\
\hline Distance modulus ....................... & $m_{B}-M_{B}=28.0$ \\
\hline Corrected absolute magnitude $(B) \ldots \ldots$. & $-14.5 \mathrm{mag}$ \\
\hline Blue luminosity $L_{B} \ldots \ldots \ldots \ldots \ldots \ldots$ & $9.4 \times 10^{7} L_{B \odot}$ \\
\hline 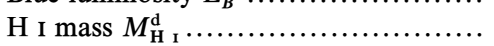 & $2.3 \times 10^{8} M_{\odot}$ \\
\hline $\mathrm{H}$ I mass-to-blue light ratio $M_{\mathrm{H}} / L_{B} \ldots$ & $2.4 M_{\odot} / L_{B \odot} \odot$ \\
\hline Dynamical mass $M_{\mathrm{dyn}} \ldots \ldots \ldots \ldots \ldots \ldots$ & $5.1 \times 10^{9} M_{\odot}$ \\
\hline
\end{tabular}

\footnotetext{
${ }^{\text {a }}$ See the discussion in $\S 2.2$.

${ }^{b}$ See the discussion in $\S 5$.

c From de Vaucouleurs et al. (1991).

${ }^{d}$ From this study.
} 
from a distance of $5.6 \mathrm{Mpc}\left(H_{0}=50 \mathrm{~km} \mathrm{~s}^{-1} \mathrm{Mpc}^{-1}\right)$ down to $2.8 \mathrm{Mpc}\left(H_{0}=100 \mathrm{~km} \mathrm{~s}^{-1} \mathrm{Mpc}^{-1}\right)$. However, at this systemic velocity the observed redshift is not yet dominated by the cosmological redshift but is affected by local effects. In order to get a feeling for this, we followed the procedure proposed by Kraan-Korteweg (1986) using the radial velocity corrected for Virgocentric infall, with a local infall velocity of $220 \mathrm{~km} \mathrm{~s}^{-1}$ and a Hubble constant of $75 \mathrm{~km} \mathrm{~s}^{-1}$ $\mathrm{Mpc}^{-1}$. This calculation puts DDO 47 at a distance of about 3.5 Mpc. If we would allow for infall toward the Great Attractor and adopt a shallow extended overdensity model, we would get a distance of $3.8 \mathrm{Mpc}$. However, the Great Attractor models imply a rather large margin of error. Given all the uncertainties, we adopt a distance toward DDO 47 of $4 \mathrm{Mpc}$, keeping in mind that this quantity is a major source of uncertainty in the discussion that follows.

\section{OBSERVATIONS}

\subsection{VLA H I Observations}

DDO 47 has been observed in the $21 \mathrm{~cm}$ line of neutral hydrogen $(\mathrm{H} \mathrm{I})$ using the $\mathrm{NRAO}^{2}$ Very Large Array (VLA) in $\mathrm{B}, \mathrm{C}, \mathrm{DnC}$, and D configurations. In total, $16 \mathrm{hr}$ were spent on-source: $2 \mathrm{hr}$ in D array, $3 \mathrm{hr}$ in DnC array, $4 \mathrm{hr}$ in $\mathrm{C}$ array, and $7 \mathrm{hr}$ in $\mathrm{B}$ array. More time was spent in the more extended arrays to partly compensate for their lower surface brightness sensitivity. Table 2 summarizes the setup of the VLA during the observations. The data reduction and analysis presented here was done using the AIPS package. $^{3}$

We flux-calibrated the data using the standard calibrator $1328+307$ (3C 286), assuming a flux density of $14.77 \mathrm{Jy}$ at $21 \mathrm{~cm}$ (according to the scale of Baars et al. 1977). The same calibrator was used for the complex bandpass corrections. The secondary phase and amplitude calibration was done using the calibrator $0735+178$, which is unresolved for the arrays used. Its flux was determined to be $2.7 \mathrm{Jy}$. DDO 47 was observed with a bandwidth of $1.56 \mathrm{MHz}$ centered at a heliocentric velocity of $265 \mathrm{~km} \mathrm{~s}^{-1}$, well outside the range of galactic $\mathrm{H}$ I emission in the direction of this object. This band was divided in 128 channels, resulting in a velocity resolution of $2.58 \mathrm{~km} \mathrm{~s}^{-1}$, after on-line Hanning smoothing.

The $u-v$ data were inspected for each array and bad data points due to either interference or cross talk between

\footnotetext{
2 The National Radio Astronomy Observatory (NRAO) is a facility of the National Science Foundation (NSF) operated under cooperative agreement by Associated Universities, Inc.

${ }^{3}$ The Astronomical Image Processing System (AIPS) has been developed by the NRAO.
}

antennae were removed, after which the data were calibrated. We Fourier transformed our B-, C-, DnC-, and D-array observations separately to assess their quality. After final editing, we combined all the data to form a single data set, which was subsequently used for mapping.

In order to remove the continuum from the line data, we first determined the line-free channels in our observation and subsequently subtracted the continuum emission in the $u-v$ plane. After that, data cubes $(1024 \times 1024$ pixels by 68 channels each) were produced using the task IMAGR in AIPS. One data cube was made with natural weighting, leading to a resolution of $15^{\prime \prime} .3 \times 15^{\prime \prime} .0$, which emphasizes large-scale structures. A second cube was produced using the ROBUST-weighting scheme (Briggs 1995). This scheme achieves the high sensitivity of natural weighting combined with a well-behaved synthesized beam, at a resolution close to that of uniform weighting. To obtain the optimum ROBUST value for our observation, the ROBUST parameter space was searched by producing maps in the sensitive regime of $-2<$ ROBUST $<2$ and inspecting each for rms noise and beam size. Eventually, we chose a value of ROBUST of 0.25 , resulting in a beam size of 7". $8 \times 7.2$ and an rms noise of $0.8 \mathrm{mJy}_{\text {beam }}^{-1}$. As it turned out, because of the excellent $u-v$ coverage, there was no need to clean the maps for sidelobes (Högbom 1974; Clark 1980). We only cleaned the maps very lightly in order to be able to calculate the correct flux in the channel maps (see the discussion in $\S 4$.1). Unless otherwise mentioned, all the results presented in the following were obtained using the uncleaned ROBUST cube.

To separate real emission from noise, we produced a socalled master cube, following the procedure explained in detail elsewhere (Walter et al. 1997). Briefly, only those regions in the full-resolution data are considered to contain emission, which in images convolved to 3-4 times the beam area (based on the natural-weighted data) contain emission above a set level (usually in the range of 2-3 $\sigma$ ) in three or more consecutive channels. These areas of genuine emission are stored as channels in the master cube. Using this cube as reference when calculating maps of the integrated properties, such as the total $\mathrm{H}$ I surface brightness map, the velocity field and the velocity dispersion map, has the advantage that the same regions are included when inspecting cubes at different resolutions and with different signal-to-noise ratios.

\subsection{Optical Observations}

Since the plates of the Palomar Sky Survey are contaminated by a reflection of a bright star in the region of interest, we obtained new $R$-band imaging of DDO 47 . The observations were made at the $2.2 \mathrm{~m}$ telescope of the Calar Alto

TABLE 2

SETUP OF THE VLA DURING THE OBSERVATIONS

\begin{tabular}{|c|c|c|c|c|c|c|c|c|}
\hline \multirow[b]{2}{*}{ CONFIGURATION } & \multicolumn{2}{|c|}{ BASELINE } & \multirow{2}{*}{$\begin{array}{c}\text { OBSERVATION } \\
\text { DATE }\end{array}$} & \multirow{2}{*}{$\begin{array}{l}\text { Total } \\
\text { Time } \\
(\mathrm{min})\end{array}$} & \multirow{2}{*}{$\begin{array}{c}\text { TOTAL } \\
\text { BANDWIDTH } \\
(\mathrm{MHz})\end{array}$} & \multirow{2}{*}{$\begin{array}{c}\text { No. of } \\
\text { ChanNELS }\end{array}$} & \multirow{2}{*}{$\begin{array}{c}\text { VeLOCITY } \\
\text { RESOLUTION } \\
\left(\mathrm{km} \mathrm{s}^{-1}\right)\end{array}$} & \multirow{2}{*}{$\begin{array}{l}\text { CENTRAL } \\
\text { Velocity } \\
\left(\mathrm{km} \mathrm{s}^{-1}\right)\end{array}$} \\
\hline & $\lambda$ & $\mathrm{km}$ & & & & & & \\
\hline В ............... & $1-54$ & $0.21-11.4$ & 1991 Dec 27 & 405 & 1.56 & 128 & 2.58 & 265 \\
\hline $\mathrm{C} \ldots \ldots \ldots \ldots \ldots$ & $0.34-16$ & $0.073-3.4$ & 1992 Mar 3 & 240 & 1.56 & 128 & 2.58 & 265 \\
\hline $\mathrm{DnC} \ldots \ldots \ldots \ldots$ & $0.166-16$ & $0.035-3.4$ & 1991 Feb 10 & 180 & 1.56 & 128 & 2.58 & 265 \\
\hline $\mathrm{D} \ldots \ldots \ldots \ldots \ldots$ & $0.166-4.9$ & $0.035-1.03$ & 1991 Apr 16 & 120 & 1.56 & 128 & 2.58 & 265 \\
\hline
\end{tabular}




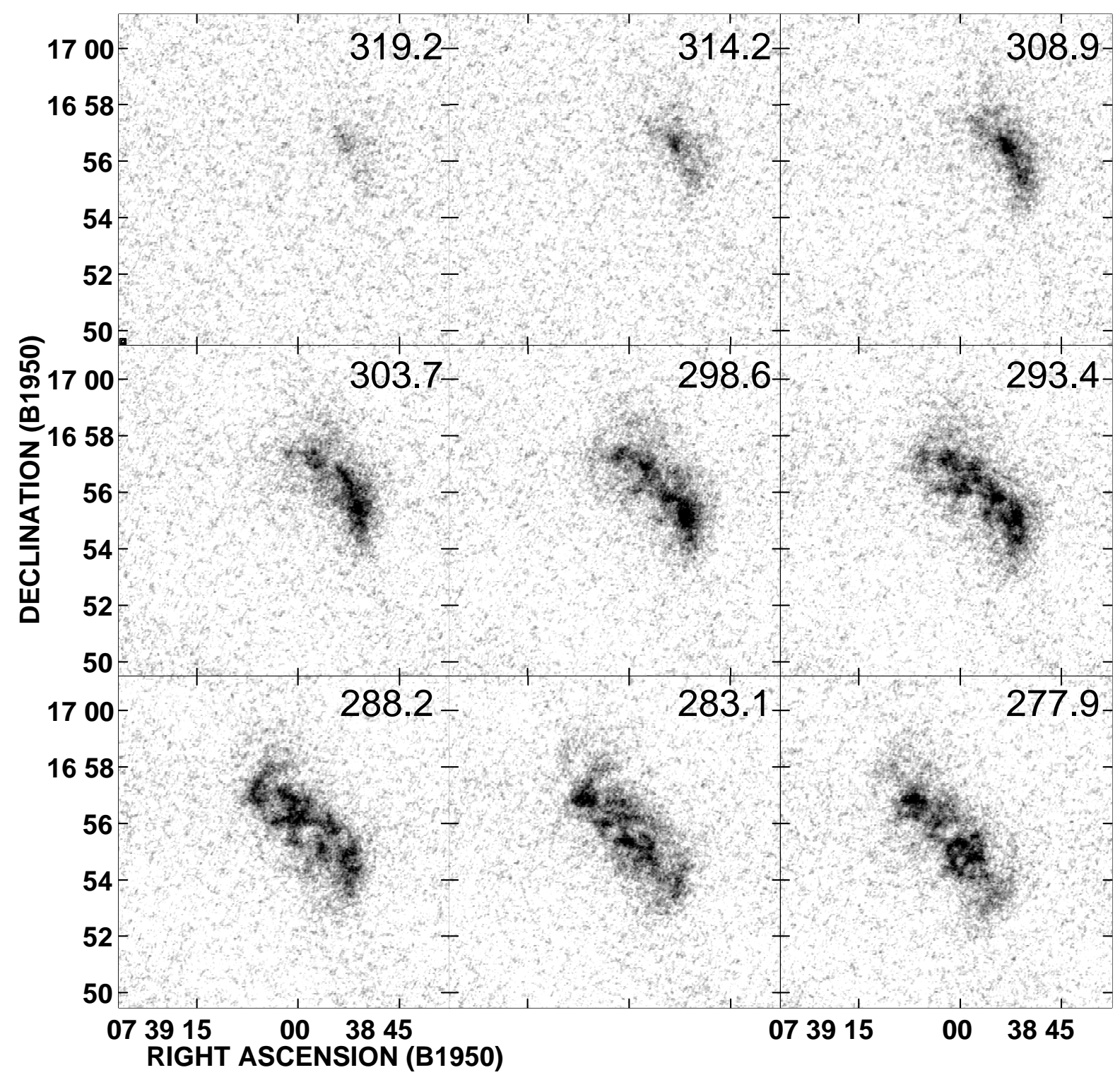

FIG. 1.-Mosaic of every other channel map of DDO 47 in which line emission was detected. The velocity in kilometers per second is plotted in the top right-hand corner of each panel. Note that the beam size $\left(7^{\prime \prime} .8 \times 7 " .2\right.$, as indicated in the box in the top left-most panel) is smaller than the resolution of this plot. Coordinates are given for the B1950.0 equinox. The gray scale is a linear representation of the $\mathrm{H}$ I surface brightness ranging from $0 \mathrm{mJy}$ beam ${ }^{-1}$ (white) to $6 \mathrm{mJy}_{\text {beam }}^{-1}$ (black).

Observatory ${ }^{4}$ in 1999 January, in Johnson $R$ band (total exposure time: $1200 \mathrm{~s}$ ). We also attempted $\mathrm{H} \alpha$ imaging; however, the quality of the resulting maps turned out to be inferior as compared with the results published by Strobel et al. (1991). We therefore decided to rely on their data concerning the $\mathrm{H} \alpha$ imaging (see $\S 7$ ). For our observations, a $2048 \times 2048$ pixel CCD was used, leading to a resolution per pixel of 0 ."5. The seeing was around 1 ".4 and the CAFOS focal reducer allowed for a field of view of radius $8^{\prime}$ to be imaged. The usual calibration steps were followed, employing the IRAF package. ${ }^{5}$ Each exposure was corrected for zero offset (bias) and was flat-fielded using sky flats. After

${ }^{4}$ The Calar Alto Observatory is operated by the Max-Planck-Institut für Astronomie (Heidelberg) jointly with the Spanish Comisión Nacional de Astronomía.

${ }^{5}$ IRAF is distributed by National Optical Astronomy Observatories, which are operated by the Association of Universities for Research in Astronomy, Inc., under contract with the NSF.
TABLE 3

GENERAL INFORMATION ON CGCG 087-033

\begin{tabular}{|c|c|}
\hline Parameters & Values \\
\hline Object $\ldots \ldots \ldots \ldots \ldots \ldots$ & CGCG 087-033 \\
\hline Right ascension $(\mathrm{B} 1950.0)^{\mathrm{a}}$ & $07^{\mathrm{h}} 39^{\mathrm{m}} 39^{\mathrm{s}} .9$ \\
\hline Declination $(\mathrm{B} 1950.0)^{\mathrm{a}} \ldots \ldots$ & $16^{\circ} 40^{\prime} 49^{\prime \prime}$ \\
\hline Adopted distance $^{\mathrm{a}} \ldots .$. & $4 \mathrm{Mpc}$ \\
\hline Scale $\left(1^{\prime \prime}\right.$ corresponds to $) \ldots \ldots \ldots \ldots$. & $19.4 \mathrm{pc}$ \\
\hline Corrected apparent magnitude $(B)^{\mathrm{b}}$.. & $15.6 \mathrm{mag}$ \\
\hline Corrected distance modulus ................ & $m_{B}-M_{B}=28.0$ \\
\hline Corrected absolute magnitude $(B) \ldots \ldots \ldots$. & $-12.4 \mathrm{mag}$ \\
\hline Blue luminosity $L_{B} \ldots \ldots \ldots \ldots \ldots \ldots \ldots \ldots$ & $1.3 \times 10^{7} L_{B \odot}$ \\
\hline 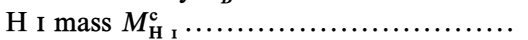 & $1.4 \times 10^{7} M_{\odot}$ \\
\hline H I mass-to-blue light ratio $M_{\mathrm{H}} / L_{B} \ldots \ldots$ & $1.1 M_{\odot} / L_{B \odot}$ \\
\hline Estimated dynamical mass $M_{\mathrm{dyn}} \ldots \ldots \ldots$ & $>1.2 \times 10^{8} M_{\odot}$ \\
\hline
\end{tabular}

${ }^{a}$ See the discussion in $\S 4.2$.

b From de Vaucouleurs et al. (1991).

${ }^{c}$ From this study. 


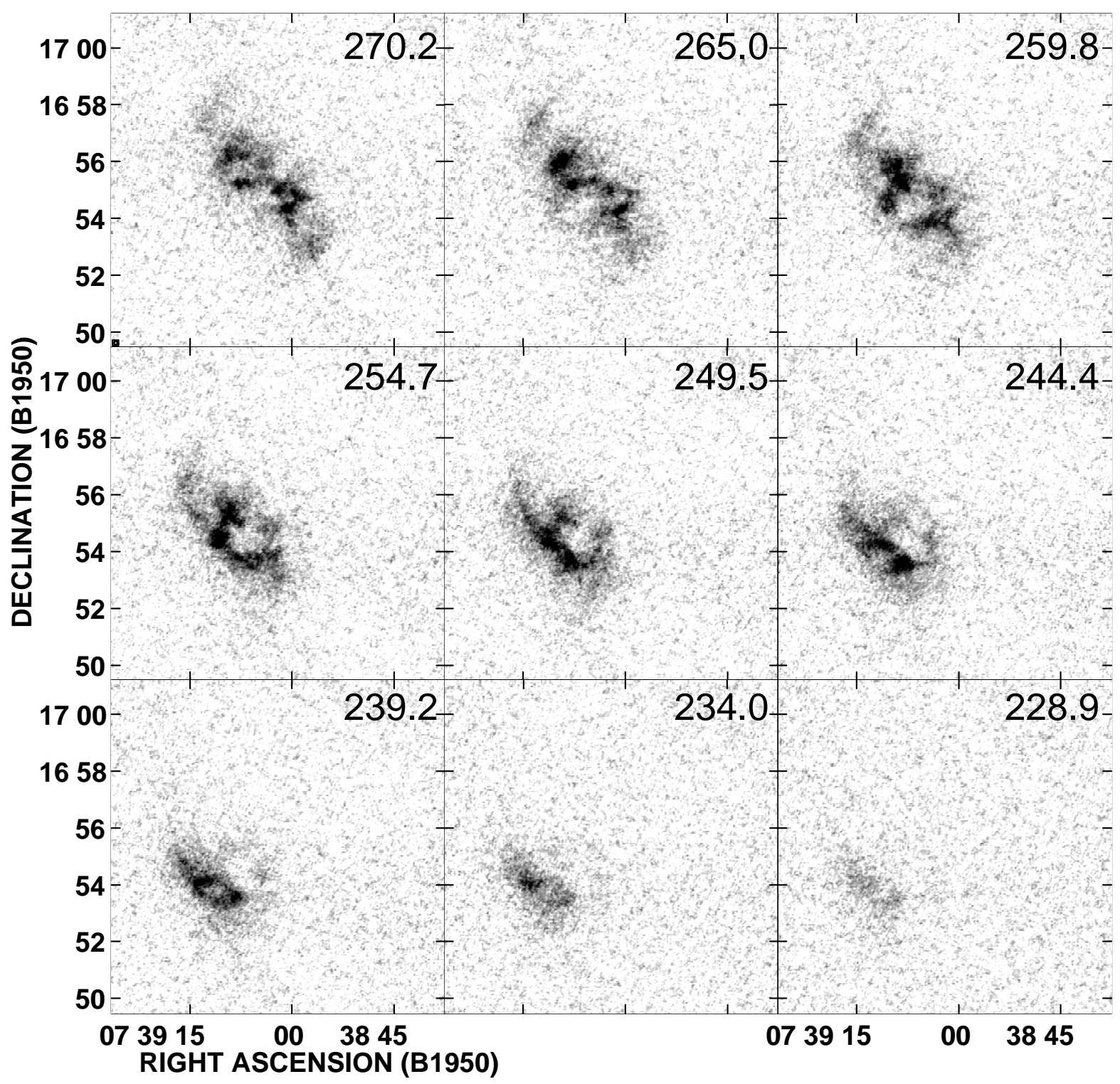

FIG. 1.-Continued

that, frames were inspected for bad pixels and shifted to align them. In the end, the exposures were combined to eliminate cosmic-ray hits and to create a final image. As it turned out, conditions were not photometric during the observing run, therefore no calibration using standard stars was performed. In order to get an approximate calibration for DDO 47, values were taken from the literature (de Vaucouleurs et al. 1983).

Coordinates for the $R$-band map were obtained using the task KOORDS in the KARMA ${ }^{6}$ software package, which computes a plate solution. We used as input an image from the Digital Sky Survey, which yielded an accuracy of better than $1^{\prime \prime}$ for our maps - adequate for our needs.

\section{H I AND OPTICAL RESULTS}

\subsection{H I Study of DDO 47}

The H I channel maps of DDO 47 are presented in Figures $1 a$ and $1 b$. Note that the beam size is too small to be

\footnotetext{
${ }^{6}$ The KARMA visualization software package was developed by Richard Gooch of the Australia Telescope National Facility.
}

properly reproduced (as indicated in the bottom left corner of the top left panel), and that only every second channel is shown to save space. The numbers in the top right indicate the heliocentric radial velocities of the $\mathrm{H}$ I gas in kilometers per second. Already at first glance, many holes, shells, and arcs are visible in the channel maps, which will be discussed in full detail in $\S 6$.

In order to get the correct $\mathrm{H}$ i flux for the channels, one has to keep in mind that the determination of $\mathrm{H}$ I fluxes of extended emission in interferometric maps is not straightforward. As explained by Jörsäter \& van Moorsel (1995), fully cleaned maps do not exist, and any cleaned map consists of the sum of two maps: one containing the restored clean components and the other, the residual map. In the former, the unit is jansky per clean beam area, and in the latter, jansky per dirty beam area. Following Walter \& Brinks (1999), we used our cleaned data cube of DDO 47 in order to calculate the real flux for our channels. We calculated this real flux $(G)$ using $G=(D \times C) /(D-R)$, where $C$ is the cleaned flux, and $R$ and $D$ are the "erroneous" residual flux and the "erroneous" dirty flux over the same area of a channel map, respectively. For a full discussion on 


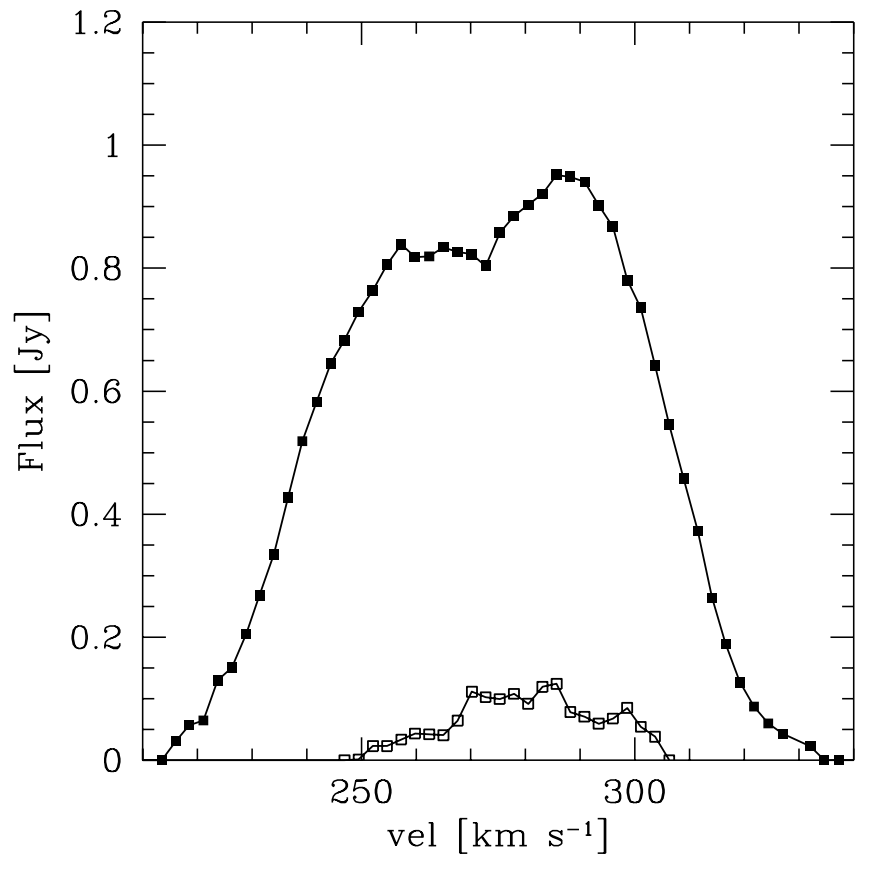

FIG. 2.-Global H I profile for DDO 47 (filled squares) and companion (open squares), obtained by integrating the channel maps over the area of the source after subtracting the continuum background. The data have been flux- and primary-beam corrected (see text). The flux is given in janksys, and the channel spacing is $2.58 \mathrm{~km} \mathrm{~s}^{-1}$.

this topic, the reader is referred to the appendix in Jörsäter \& van Moorsel (1995). We followed this procedure for every single channel to obtain the global $\mathrm{H}$ I profile that is presented in Figure 2.

From this graph, we infer a systemic velocity of DDO 47 of $273 \pm 2 \mathrm{~km} \mathrm{~s}^{-1}$. The total integrated flux is $61 \mathrm{Jy} \mathrm{km} \mathrm{s}^{-1}$, corresponding to a total $\mathrm{H}$ I mass of $2.3 \times 10^{8} M_{\odot}$ ' (adopting a distance of $4 \mathrm{Mpc} ; \S 2.2$ ). To check how much flux we missed because of the missing of short spacings in the interferometer observations, we compare this value with the one published by Hoffman et al. (1996). They mapped DDO 47 with the Arecibo single-dish telescope and derived a total flux of $67 \mathrm{Jy} \mathrm{km} \mathrm{s}^{-1}$, in good agreement with our VLA observation. However, as will be discussed in $\S 5$, the fact that we recover almost all flux with our VLA observations does not imply that we fully recover all large-scale structures.

The high-resolution $\mathrm{H}$ I surface density map of DDO 47 is presented in Figure 3. It was obtained by adding all channels after blanking with the master cube. The size of the beam is plotted in the bottom left of the map. We are viewing DDO 47 almost face-on. Its interstellar medium is obviously dominated by several holes (see the discussion in $\S 6$ ). In addition, there is some indication for spiral structure at large radii.

The deprojected $\mathrm{H}$ I surface density of DDO 47 , averaged in annuli, and corrected to face-on view, is shown in Figure 4. An inclination of $35^{\circ}$ was adopted for the deprojection (see $\S 5$ ). Note that most of the structure in this plot, especially at small radii, is due to the large holes in the neutral ISM of DDO 47.

The velocity field of DDO 47 was determined by calculating the first moment (intensity-weighted mean velocity) and is presented in Figure 5. Since the high-resolution velocity field is dominated by small-scale motions within the ISM, the naturally weighted cube was convolved to $30^{\prime \prime}$ to emphasize the global characteristics of the velocity field. The underlying gray scale is a linear representation of the $\mathrm{H}$ I surface brightness map at full resolution (as indicated by the beam size). Note that the isovelocity contours are almost parallel out to the last measured point, which indicates that we have not yet reached the flat part of the rotation curve (which is common for dwarf galaxies). The dynamics of DDO 47 will be discussed in more detail in $\S 5$.

\subsection{CGCG 087-033: The Companion of DDO 47}

Searching for companions around dwarf galaxies is important, since gravitational interactions between dwarfs, like interactions between larger galaxies, are believed to trigger star formation. Surveys to identify possible companions around BCD galaxies and low surface brightness (LSB) dwarf galaxies have been undertaken, e.g., by Telles $\&$ Terlevich (1995), Campos-Aguilar, Moles, \& Masegose (1993), and Telles \& Maddox (2000). These authors claim that there is no indication for interactions with (bright) companion objects. However, Taylor (1997) finds that dwarf galaxies that are experiencing a burst of star formation tend to have low-mass companions, whereas nonstarbursting (LSB) dwarfs show a much lower frequency of companions, lending support to the picture that interactions can provide the trigger.

In order to find out whether DDO 47 (which is not in a starbursting phase) has a companion dwarf galaxy, we mapped an area as large as possible around DDO 47. Interestingly, we indeed detected a likely companion galaxy at a projected distance of $17^{\prime}$ (corresponding to a linear distance of about $20 \mathrm{kpc}$; see Fig. 6) and at approximately the same systemic velocity.

The companion clearly is not an artifact and is present in maps obtained from the individual array configurations, i.e., before combining them, except for the B-array observations, for which the signal-to-noise ratio was insufficient to detect the companion. The maps of the companion that are presented in the following are based on the combined D-, DnC-, and C-array observations only, using natural weighting (to recover as much extended emission as possible), were cleaned down to 3 times the noise level in a singlechannel map and were primary-beam corrected. In order to separate $\mathrm{H}$ I emission from the noise, we again produced a master cube, as described above ( $\S 3.1)$. Figure 6 is the result of the data reduction and shows the relative position of the two galaxies (based on the $\mathrm{D}, \mathrm{DnC}$, and $\mathrm{C}$ data only for both objects; see discussion below). The response of the primary beam at this distance is less then half of the response at the pointing center. It should be noted that had we not searched especially for it, we would not have detected the companion.

Figure 7 shows the channel maps of the companion. The maps have been convolved to $45^{\prime \prime}$ to enhance the sensitivity to extended structures. The numbers in each panel again indicate the radial velocity of the $\mathrm{H}$ I gas and the beam size is indicated in the bottom left-hand corner of the top leftmost panel. Emission was found in 21 consecutive channels. Note that the center of emission is shifting in the channels from west to east, indicating the sense of rotation of the companion. A possible elongation toward the south of the companion is also visible in quite a few channel maps. If this feature is real, it might be a tidal arm, which would indicate an ongoing or past interaction with the parent galaxy. 


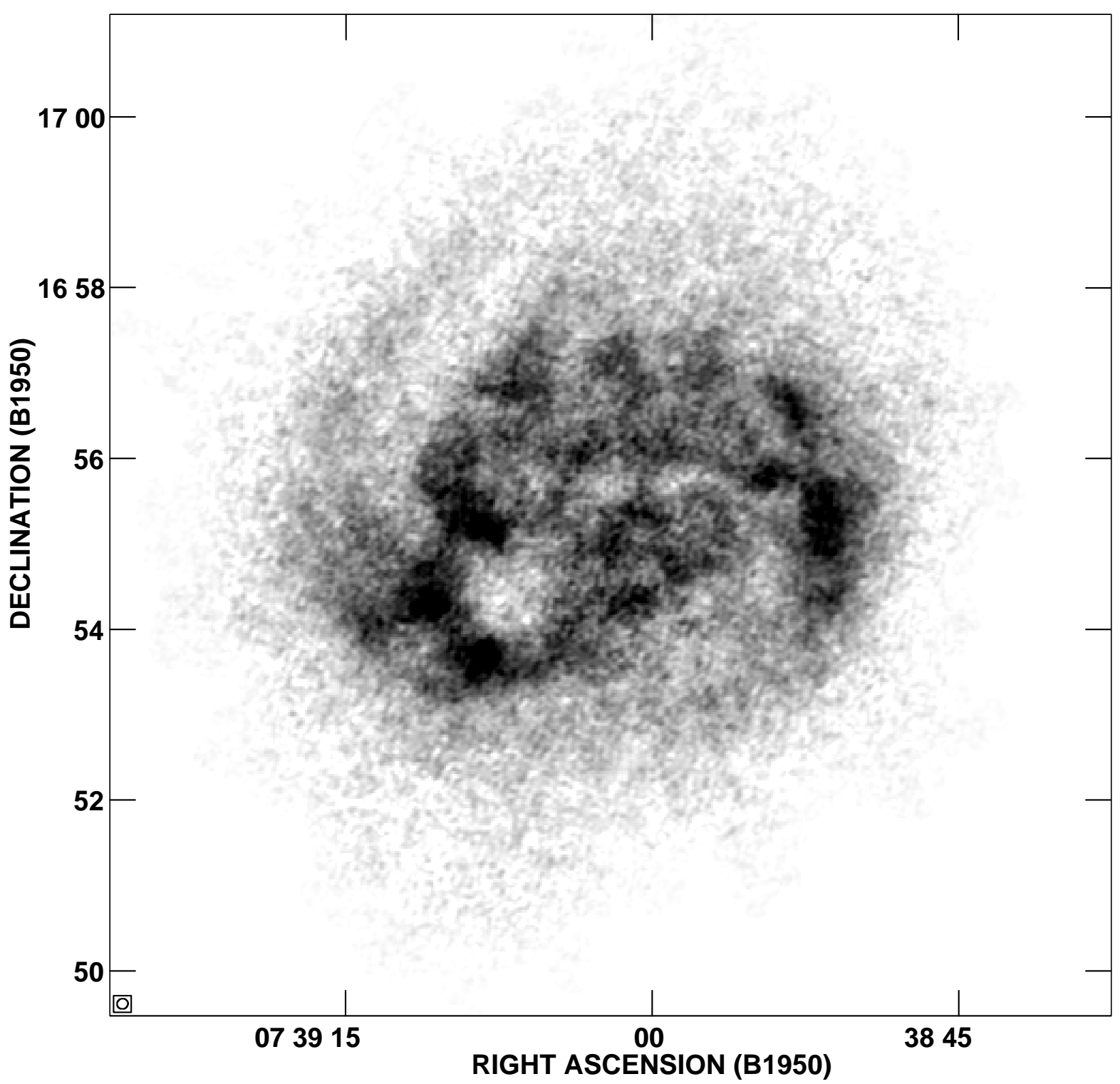

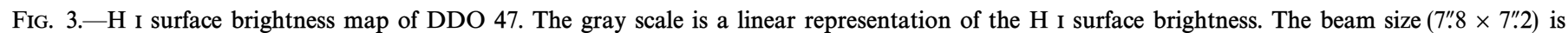
indicated in the bottom left.

The $\mathrm{H}$ I profile of the companion (flux- and primarybeam corrected) is presented in Figure 2. Its systemic velocity of $283 \pm 3 \mathrm{~km} \mathrm{~s}^{-1}$ is almost identical to that of DDO 47 . This suggests that both galaxies are moving probably in the plane of the sky, and the observed projected distance is in fact close to the real one. The total flux integral of the companion is $3.85 \mathrm{Jy} \mathrm{km} \mathrm{s}^{-1}$, which corresponds to a total $\mathrm{H}$ I mass of $1.4 \times 10^{7} M_{\odot}$, assuming, as for DDO 47 , a distance of $4 \mathrm{Mpc}$.

The velocity field of the companion CGCG 087-033, superposed on its $\mathrm{H}$ I surface density map, is presented in Figure 8 . The beam size shows that we indeed resolve the object, and that the companion is rotating. The major axes of the two galaxies are almost perpendicular to each other; their sense of rotation is the same. The data do not allow any detailed model fitting to the velocity field. Nonetheless, one can estimate the dynamical mass by simply measuring the full width at zero intensity of the velocity profile, either based on Figure 2 or Figure 7, from which we derive a value of $50 \mathrm{~km} \mathrm{~s}^{-1}$. The total extent of the object is $1.1 \mathrm{kpc}$. The inclination is unknown, and neither the optical image nor the $\mathrm{H}$ I distribution are of any particular help in determining or restricting its value. Hence, we can only determine a lower limit for the dynamical mass of CGCG 087-033, which we estimate at $M_{\text {dyn }}>1.2 \times 10^{8} M_{\odot}$. This implies that the likely companion to DDO 47 contains a considerable amount of dark matter.

\subsection{Comparison with Optical Data}

Figure 9 shows an overlay of our $R$-band image with the $\mathrm{H}$ I surface density map of DDO 47 . The presence of the many foreground stars illustrates the low galactic latitude of DDO $47\left(b=-4^{\circ}\right)$. The apparent corrected blue magnitude of DDO 47 is 13.5 mag (extinction corrected for absorption due to the Milky Way, according to de Vaucouleurs et al. 1991), which corresponds to an absolute blue magnitude of -14.9 mag. DDO 47 therefore falls well within the definition of dwarf galaxies (usually defined as being objects less luminous than an absolute blue magnitude of $-18 \mathrm{mag}$ ). Converting this blue magnitude to blue 


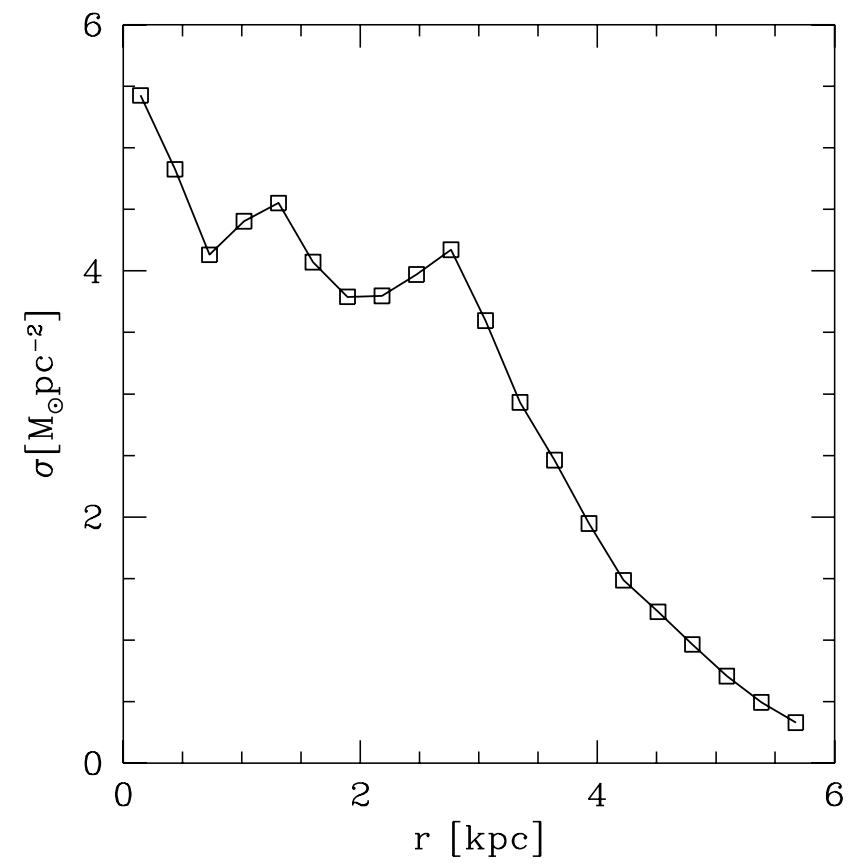

FIG. 4.- - H I surface density of DDO 47 in units of $M_{\odot} \mathrm{pc}^{-2}$, obtained by averaging in annuli after deprojecting for an inclination of $35^{\circ}$ and reducing the results to face-on values.

solar luminosity using $M_{B \odot}=5.43$, we find a value of $9.4 \times 10^{7} L_{B \odot}$. The H I mass-to-blue light ratio of DDO 47 is $M_{\mathrm{H} \mathrm{I}} / L_{B}=2.4 M_{\odot} / L_{B \odot}$.

At the position of the companion, relatively concentrated optical emission is present on the POSS plates. To investigate this further, we obtained new optical imaging of this region of the sky using the Calar Alto $2.2 \mathrm{~m}$ telescope. Figure 10 is an overlay of the total $\mathrm{H}$ I map with the optical image. The companion galaxy had previously been cata-

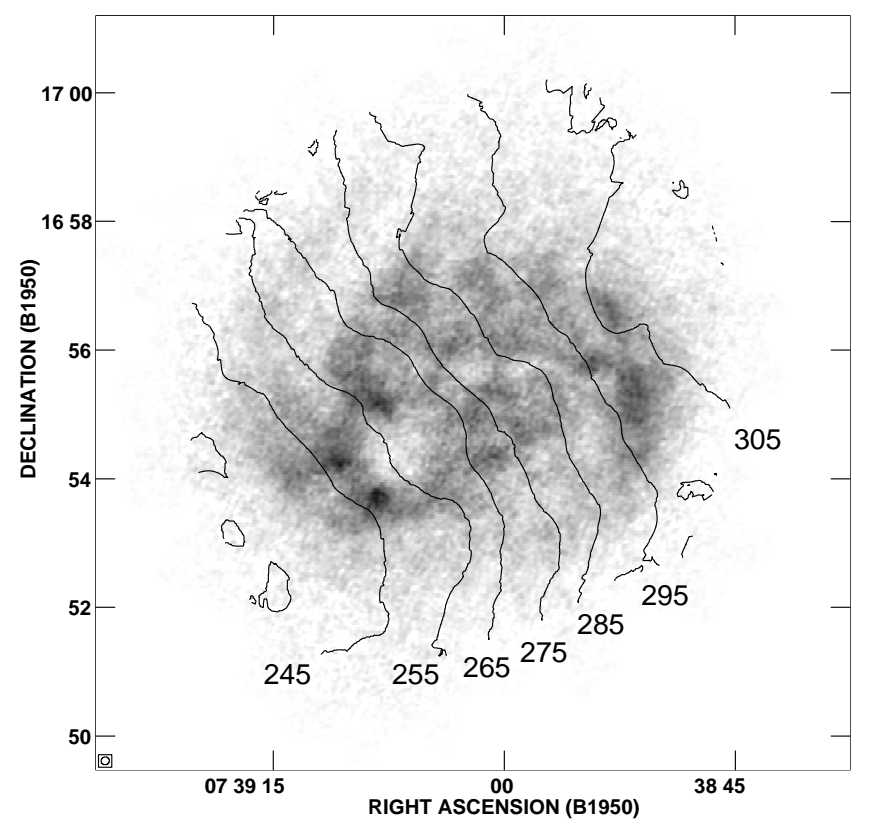

FIG. 5.- Isovelocity contours of DDO 47 based on data convolved to $30^{\prime \prime}$. The velocities are given in kilometers per second. The gray-scale image is a linear representation of the $\mathrm{H}_{\mathrm{I}}$ surface brightness map at full resolution.

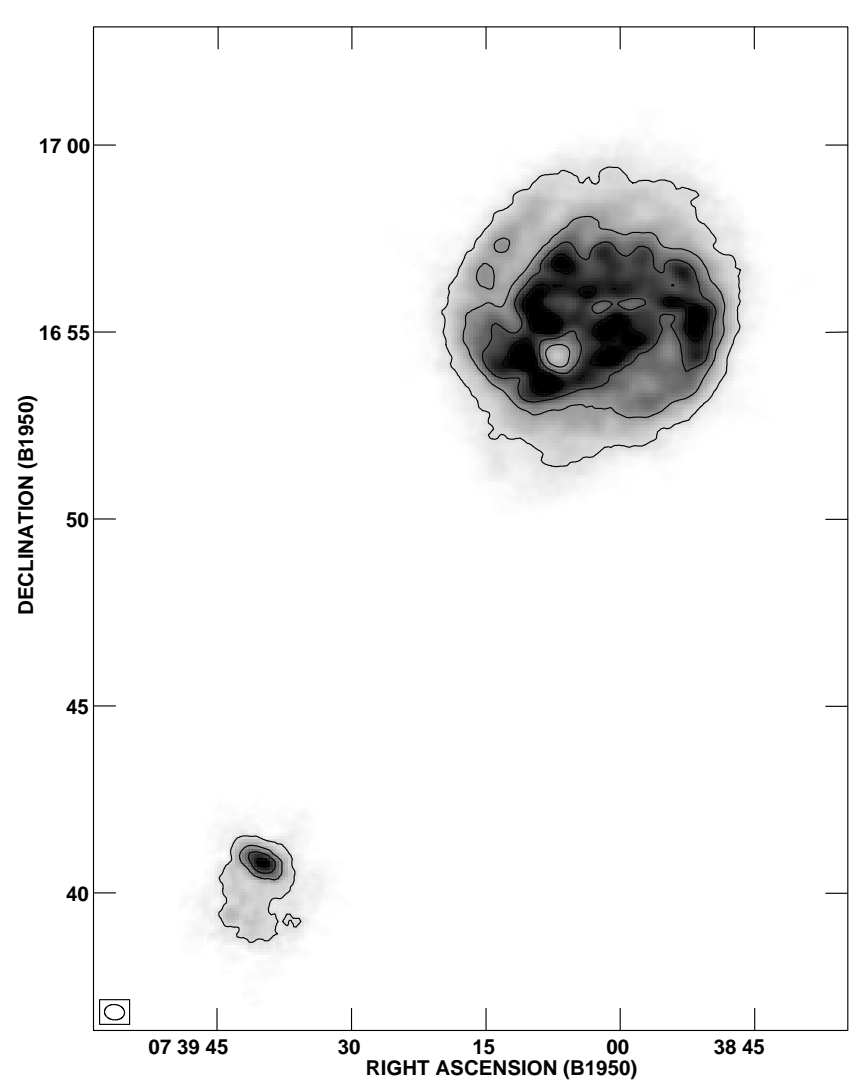

FIG. 6.-H I surface brightness map of DDO 47 and CGCG 087-033, showing the relative positions of these two galaxies. The linear projected separation of the two objects is $20 \mathrm{kpc}$ (assuming a distance of $4 \mathrm{Mpc}$ ). The gray scale and contours are linear representations of the (primary-beam corrected) $\mathrm{H}$ I surface brightness map. Contours are shown at 1.2, 3.6, 6.0, 8.4 , and $10.8 \times 10^{20} \mathrm{~cm}^{-2}$

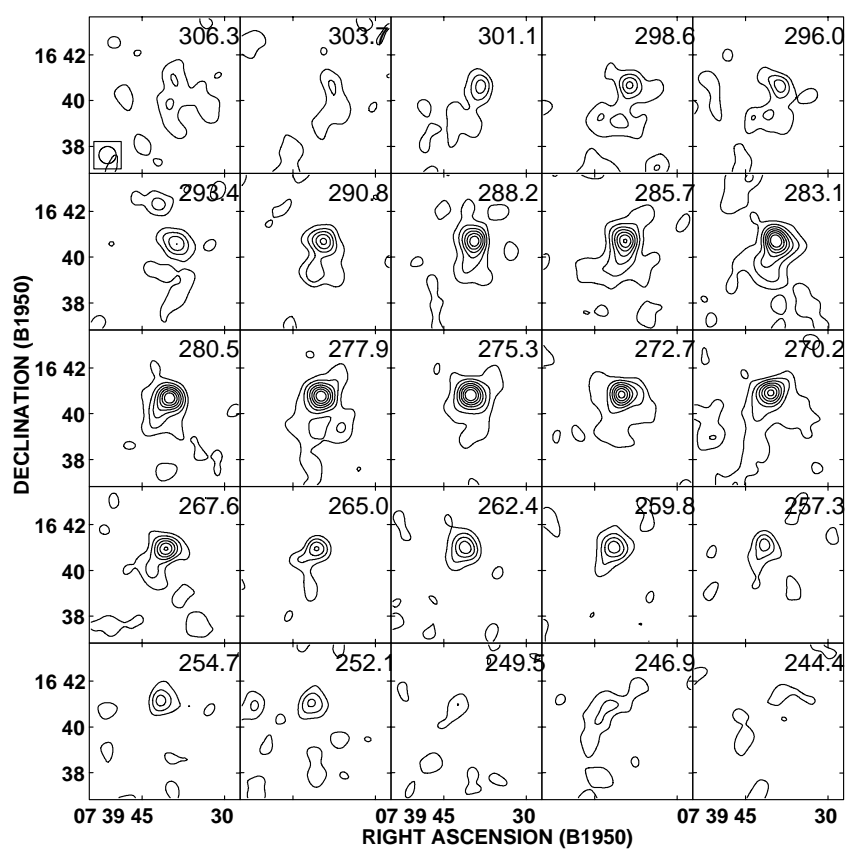

FIG. 7.-Mosaic of the channel maps of CGCG 087-033 in which line emission was detected. The size of the beam $\left(45^{\prime \prime}\right)$ is indicated in the top left-most panel. The velocity of each channel in kilometers per second is plotted in the right-hand corner. Contours are shown at 0.6, 1.2, 2.4, 3.6, $4.8,6.0$, and $7.2 \mathrm{~K}$. 


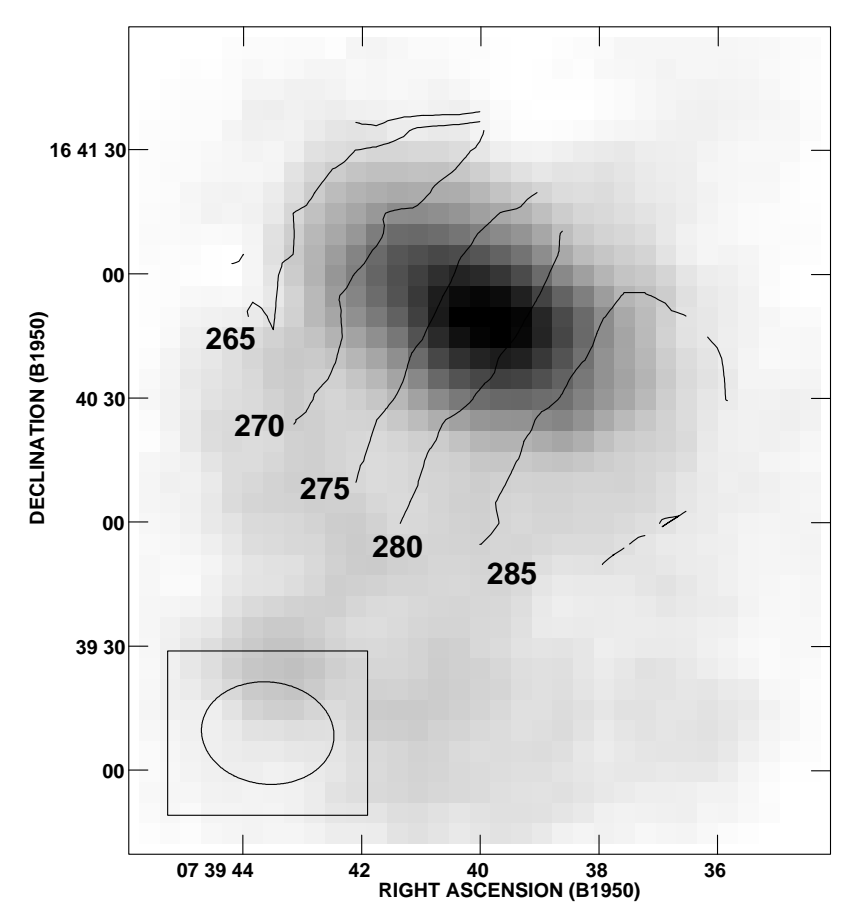

FIG. 8.-H I surface brightness map (gray scale) and isovelocity contours of the companion CGCG 087-033. The velocities are given in kilometers per second. The beam size $\left(32^{\prime \prime} \times 25^{\prime \prime}\right)$ is indicated in the bottom left.

loged as CGCG $087-033$ and has recently been classified as a new nearby dwarf galaxy candidate (Karachentseva \& Karachentsev 1998). It has a blue magnitude of 15.6 mag. Assuming that this galaxy is at the same distance as DDO 47 , its total blue magnitude is about $-12.4 \mathrm{mag}$, which leads to a luminosity of $1.3 \times 10^{7} L_{B \odot}$. The $\mathrm{H}$ I mass-toblue light ratio is $M_{\mathrm{H}} / L_{B}=1.1 M_{\odot} / L_{B \odot}$. A summary of the derived properties of CGCG 087-033 can be found in Table 3.

\section{DYNAMICAL STUDY OF DDO 47}

\subsection{Dark Matter Content of DDO 47}

In the following, we will investigate whether dark matter is needed to explain the overall dynamics of DDO 47 . We will focus our analysis on the rotation curve of DDO 47. A full mass decomposition, as well as dynamical modeling, is beyond the scope of this paper.

To derive its rotation curve, the task ROTCUR in the GIPSY $^{7}$ package was used (as described by Begeman 1989). This task uses the fact that the projected circular velocity, $v_{c, p}$, of an object is related to the true rotational velocity, $v_{c}$, assuming circular orbits of the gas via

$$
v_{c, p}(\Theta, i, r)=v_{\text {sys }}+v_{\mathrm{c}}(r) \sin i \cos \left(\Theta-\Theta_{0}\right),
$$

where $v_{\text {sys }}$ is the systemic velocity, $i$ is the inclination, $\Theta_{0}$ is the position angle of the receding major axis (also referred

\footnotetext{
${ }^{7}$ The Groningen Image Processing System (GIPSY) is distributed by the Kapteyn Astronomical Institute, Groningen, Netherlands.
}

to as P.A.), and $\Theta-\Theta_{0}$ is the angle measured with respect to the receding major axis of the object. Basically, a set of these parameters that best represents the observed velocity field at all radii must be found by fitting annuli.

We took for the input velocity field (first-moment map) a map derived on the basis of a cube smoothed to a resolution of $30^{\prime \prime}$. We did this in order to smear out small-scale structures, such as those due to the holes, that are not relevant for a dynamical analysis of the galaxy as a whole.

We then proceeded as follows: First, using best guesses for the systemic velocity $v_{\text {sys }}$ and the kinematic center of the object, a fit for the inclination $i$, the position angle P.A., and an approximate rotation curve were calculated. Once fits for $i$ and P.A. as a function of radius were found, these values were fixed, and $v_{\text {sys }}$ and the center positions were fitted. We repeated this procedure until consistent values for the parameters were found. After that, a final rotation curve was fitted to the velocity fields.

Unfortunately, ROTCUR did not give stable results for the inclination $i$. This is no wonder really, since the inclination is rather small (as can already be seen from the firstmoment map) and hence difficult to determine. Moreover, the rotation curve shows no sign of turning over, confirming that the galaxy is rotating like a solid body out to the last measured point. We therefore decided to set the inclination to $35^{\circ}$, an average value of the fitting process that is in agreement with the projected shape of the first-moment map (assuming an axis ratio of $q=b / a=0.1$ ).

With the inclination fixed, the model parameters that we determined in the course of the rotation curve fitting are as follows: systemic velocity, $v_{\text {sys }}=272 \mathrm{~km} \mathrm{~s}^{-1}$; inclination (fixed), $i=35^{\circ}$; and average position angle, P.A. $\approx 310^{\circ}$. The final adopted rotation curve is presented in Figure 11. All points presented here are independent measurements (with a separation of $30^{\prime \prime}$, i.e., the width of the convolved beam). The rotation curve is plotted in the bottom panel, whereas the top panel shows the change of position angle, P.A., with radius. The squares are the solutions using the full velocity field; the up and down triangles indicate the results using the receding and approaching sides of the galaxy only, respectively. The deviations of these values from those based on the full velocity field are probably the best estimate for the typical error in the determination of the rotation curve. Note that the rotation curve based on the VLA data shows solid-body rotation throughout, with perhaps some flattening at larger galactocentric radii-a common feature in dwarf galaxies. The position angle changes with radius - an effect that is already visible "by eye" from the velocity field. One should keep in mind, however, that the inclination has been fixed to a constant value.

Using the results by Hoffman et al. (1996), who also derived a rotation curve for DDO 47, we can combine our dynamical analysis with their results (Fig. 12). Note that they traced the rotation curve to substantially larger values with the Arecibo telescope than we can with our VLA data. At first glance, this is unexpected, since our flux analysis shows that we hardly seem to miss any flux, retrieving of the order of $90 \%$ of the Arecibo value. In order to check where we might be missing some low-intensity, extended structure, we artificially convolved our data to the resolution of the Arecibo observations. This confirmed that we did not recover $\mathrm{H}$ I emission at large radii with the VLA interferometer. This can be attributed to the fact that even the most 


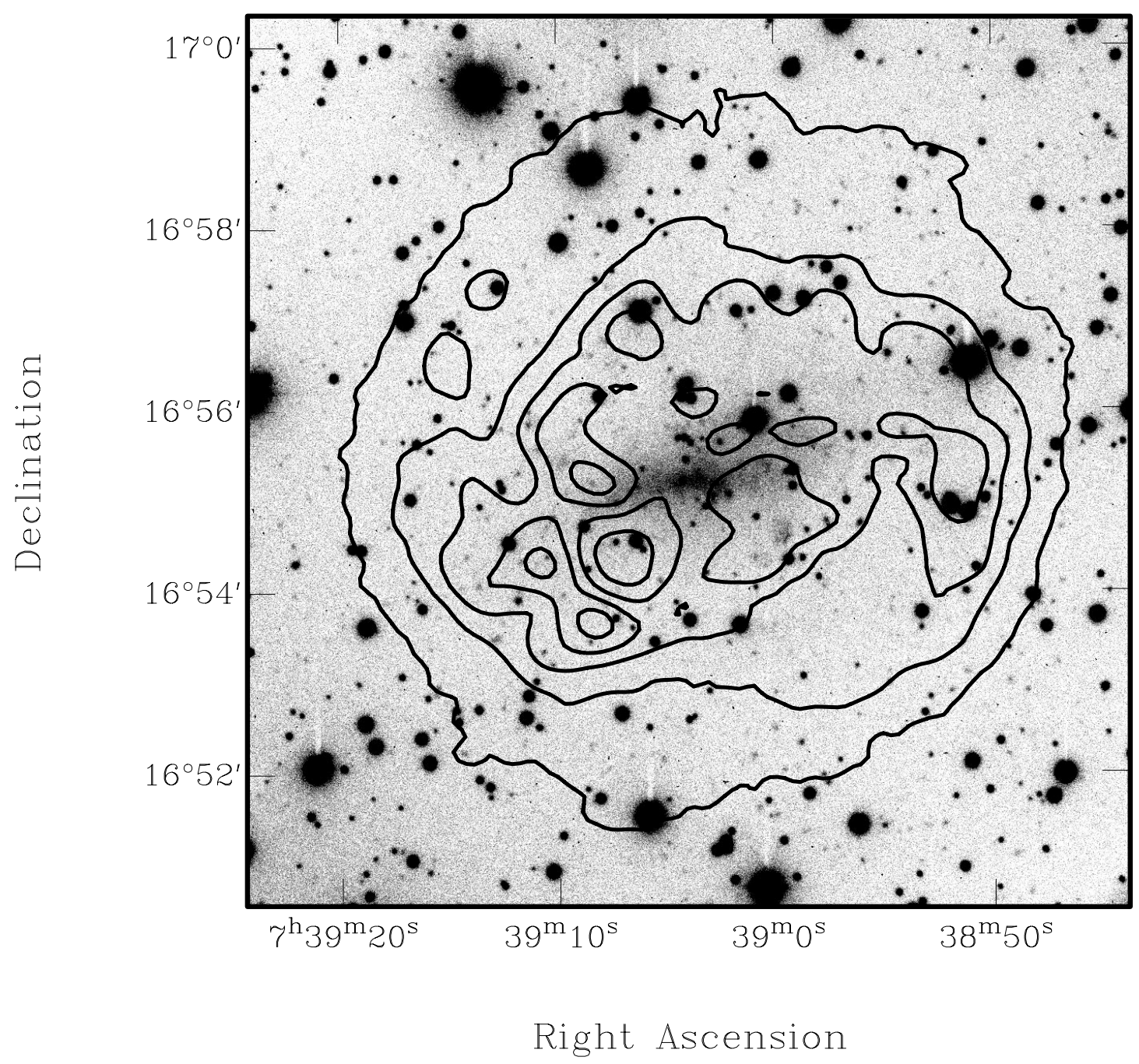

FIG. 9. $-R$-band image of DDO 47 obtained with the $2.2 \mathrm{~m}$ telescope of the Calar Alto Observatory. The overlaid contours represent the total $\mathrm{H}$ i surface brightness map as shown in Fig. 6. Contours are shown at 1.2, 3.6, 6.0, 8.4, and $10.8 \times 10^{20} \mathrm{~cm}^{-2}$.

compact $\mathrm{D}$ array is not sensitive to structures larger than $9^{\prime}$. Interestingly, Hoffman et al. (1996) find that diameters of dwarf galaxies measured with the Arecibo telescope are always larger than what is found by radio synthesis observations, even though the total flux measured by Arecibo is only a few percent larger. From their data, it appears that the galaxy shows a flat rotation curve for larger radii, indicating that we are only able to recover the rising part of the rotation curve with our VLA observations. Their innermost point is probably affected by an inaccurate central position, as well as by beam smearing.

Adopting an inclination of $35^{\circ}$ and including the results of Hoffmann et al. (1996), we derive a dynamical mass out to the last measured point of the rotation curve of DDO 47 of $M_{\mathrm{dyn}}=5.1 \times 10^{9} M_{\odot}\left(v_{\max }=54 \mathrm{~km} \mathrm{~s}^{-1}\right.$, and $r_{\max }=7.5$ $\mathrm{kpc})$. In order to determine a value for the total gas mass, one has to correct for the contribution of primordial Helium; we adopted a correction factor of 1.35 . For lack of good data, we decided not to attempt a correction for ionized or molecular hydrogen. The adopted mass for the gas content of DDO $47, M_{\text {gas }}=3.1 \times 10^{8} M_{\odot}$, is therefore a lower limit. The stellar mass contribution is difficult to estimate, since the mass-to-light ratio of the stars in the galaxy is unknown. We decided to adopt a solar mass-to- light ratio of $M_{*} / L_{B}=1.0 M_{\odot} / L_{B \odot}$. This is in line with what we derived for another dwarf galaxy, II Zw 33 (Walter et al. 1997), and in agreement with the range of values that were obtained by Broeils (1992; see also Stil 1999) for a sample of galaxies with Hubble type later than Sd. Following this approach, this yields a stellar mass component of $9.4 \times 10^{7} M_{\odot}$.

In summary, the total observed mass of the gas and the stars in DDO 47 is therefore $\approx 4 \times 10^{8} M_{\odot}$, an order of magnitude lower than the dynamical mass of $\approx 5 \times 10^{9}$ $M_{\odot}$. This indicates that DDO 47 is yet another dwarf galaxy, whose dynamics are clearly dominated by dark matter.

\subsection{Thickness of the $\mathrm{H}$ I Disk}

Fairly recently, it has been shown that the thickness of the $\mathrm{H}$ I layer of dwarf galaxies is much larger than that found in more massive spirals. Brinks \& Burton (1984) derived a scale height for the $\mathrm{H}$ I in M31 of about $100 \mathrm{pc}$, whereas in dwarf galaxies like Ho II (Puche et al. 1992), IC 2574 (Walter \& Brinks 1999), NGC 6822 (de Blok \& Walter 2000), or Ho I (Ott et al. 2001), this value is several times higher (see also Staveley-Smith et al. 1992). Note that since dwarf galaxies are smaller in linear size, the relative 


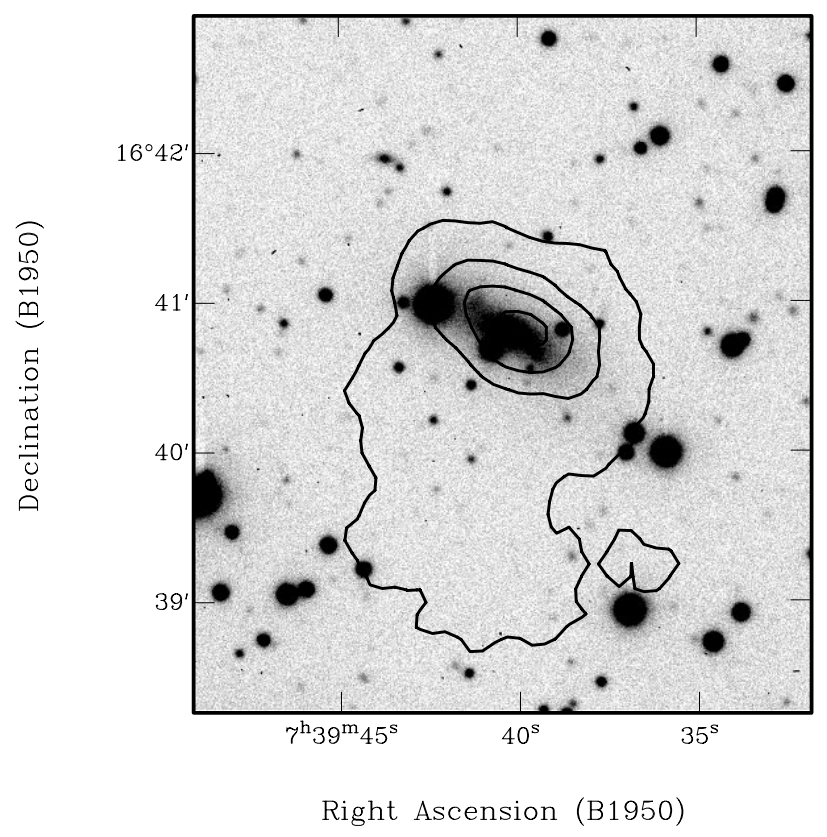

FIG. 10. - Same as Fig. 9, but for CGCG 087-033. Contours are shown at $1.2,3.6,6.0$, and $8.4 \times 10^{20} \mathrm{~cm}^{-2}$.

thickness of their $\mathrm{H}$ I layer or aspect ratio is therefore even larger.

To determine the thickness of the $\mathrm{H}$ I layer in the case of DDO 47, we follow the arguments given by Puche et al. (1992) and use the velocity dispersion of the $\mathrm{H}$ I gas in quiescent parts of the galaxy to determine its scale height. The idea behind this is that the scale height should be proportional to the velocity dispersion of the gas and inversely proportional to the square root of the mass density in the disk (see also the discussion in Ott et al. 2001). By taking

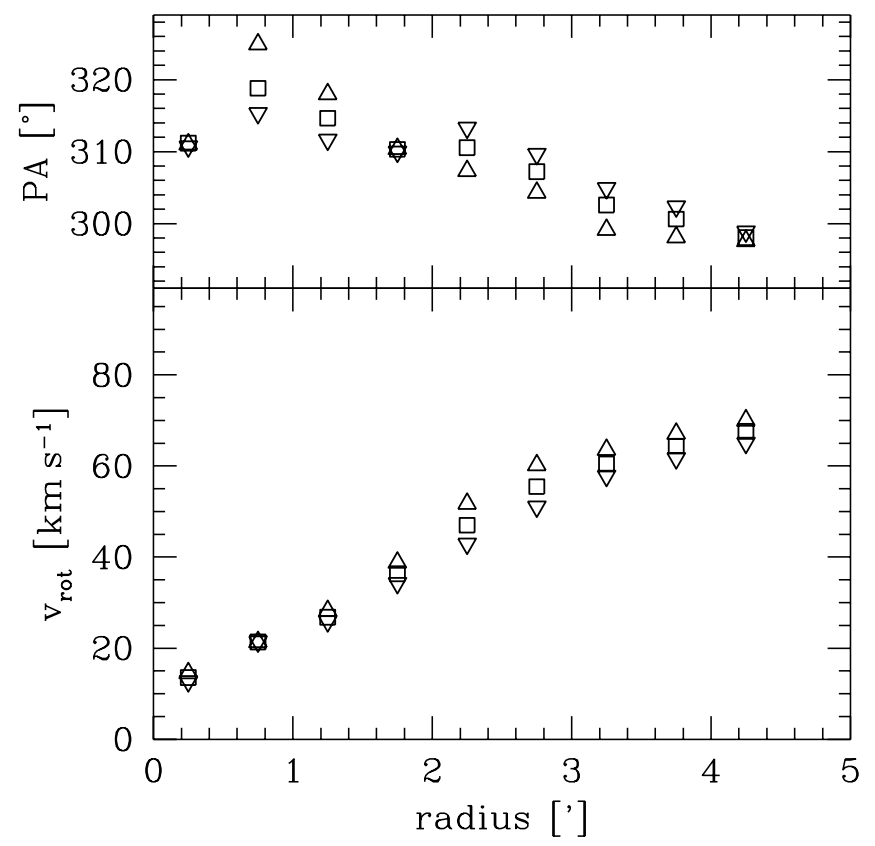

FIG. 11.-Derived rotation curve for DDO 47 (bottom). The top panel shows the change in the position angle for a fixed inclination. The different symbols represent fitting results for the approaching (down triangle), receding (up triangle), and both halves (squares) of the velocity field.

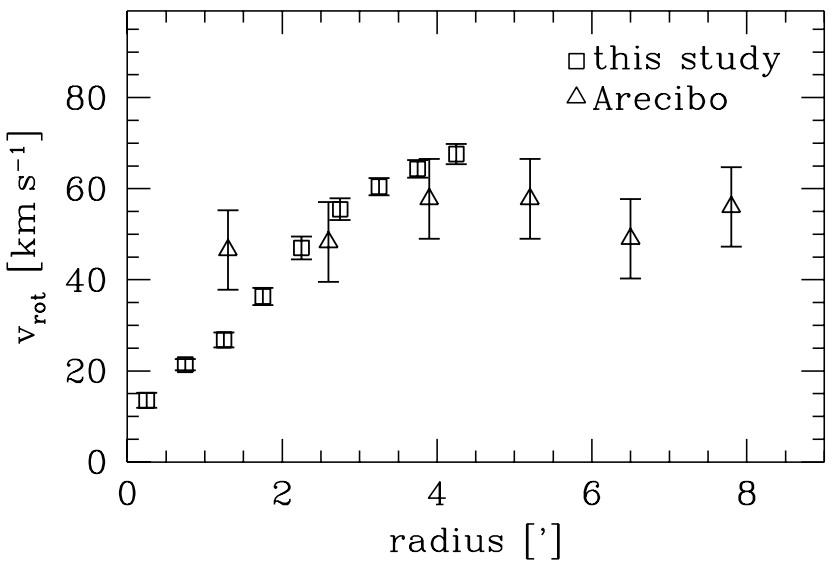

FIG. 12.-Final adopted rotation curve for DDO 47. The squares are the values derived from our data; the up triangles show the values determined by Hoffman et al. (1996) using the Arecibo telescope.

several spectra through the ROBUST-weighted $\mathrm{H}$ I cube, we derive a velocity dispersion of $8 \mathrm{~km} \mathrm{~s}^{-1}$ in the quiescent parts of DDO 47. To get an approximate mass volume density near the plane of DDO 47, we first divide the total dynamical mass by the volume of the galaxy (assuming a spherical distribution). This gives an average volume density of $0.12 \mathrm{~cm}^{-3}$, which we then multiply by a factor of 3 to get an approximate value for the volume density in the plane of the galaxy. With this value, we derive a $1 \sigma$ thickness of the $\mathrm{H}$ I layer of about $500 \mathrm{pc}$-comparable to what is found in other dwarf galaxies.

\section{H I HOLES AND SHELLS IN DDO 47}

From the channel maps (see Fig. 1), it is already clear that many features in the form of holes shape the global appearance of the ISM in DDO 47. In the standard picture, these features are due to the combined effects of stellar winds and

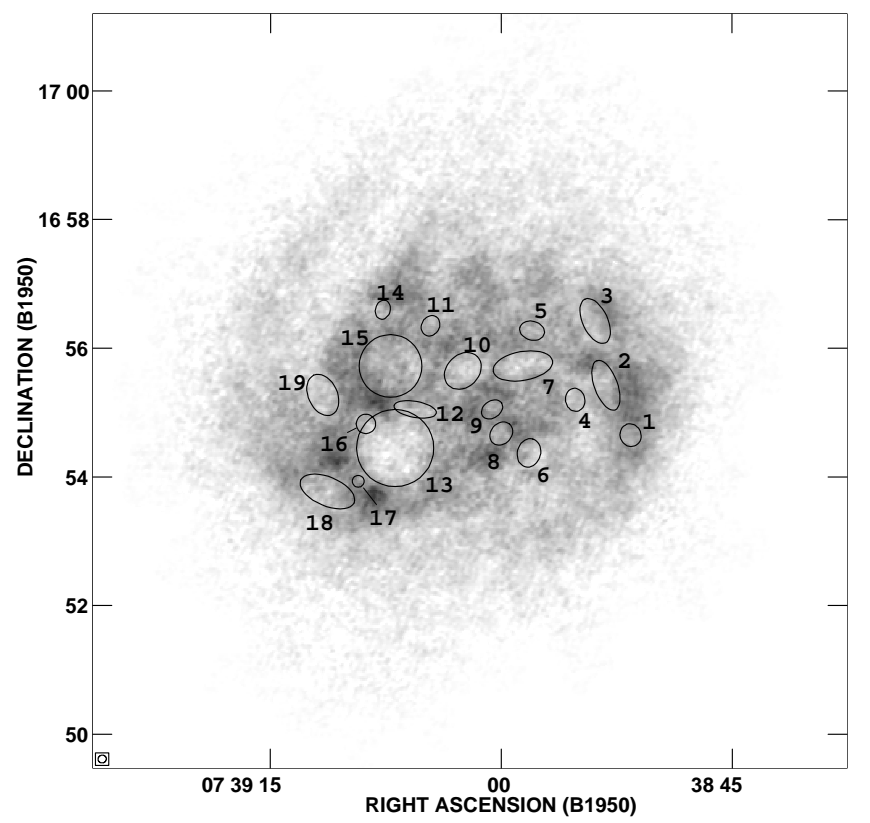

FIG. 13.- Location of the $\mathrm{H}$ I holes found in DDO 47 (with sequence numbers). The gray-scale map is a linear representation of the $\mathrm{H}$ i surface brightness map. 


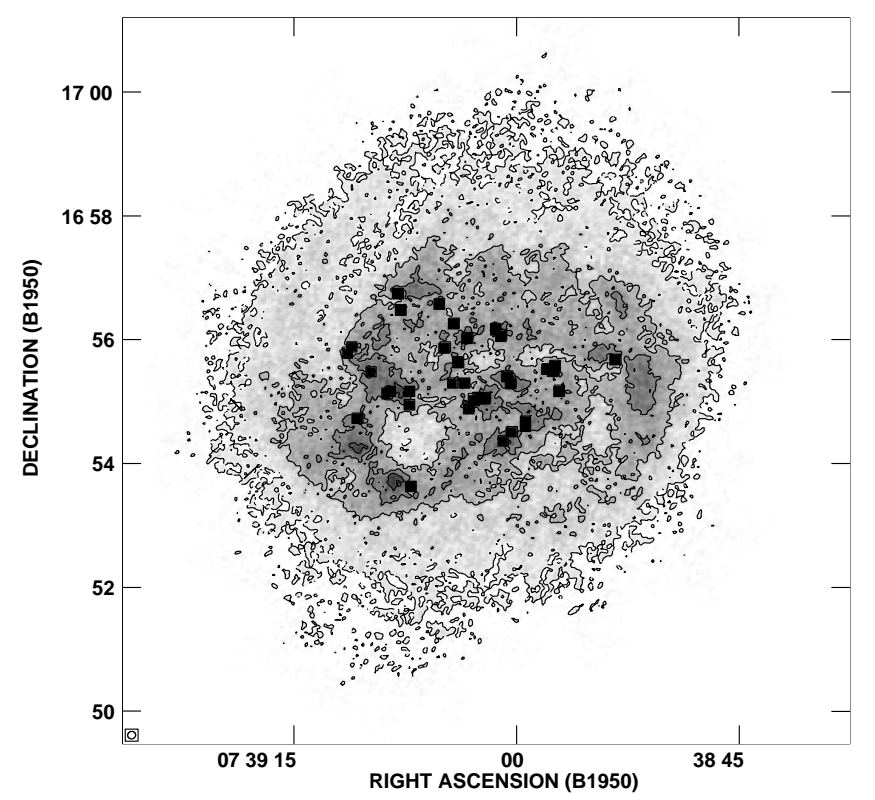

FIG. 14.- Relative location of the $\mathrm{H}$ II regions in DDO 47, detected by Strobel et al. (1991; filled squares) with respect to the H I distribution. The gray scale is a representation of the $\mathrm{H}$ I surface brightness distribution. The contours represent $\mathrm{H}$ I column densities of $1,5,9$, and $14 \times 10^{20} \mathrm{~cm}^{-2}$.

supernova explosions within young star-forming regions (see Stewart \& Walter 2000 for a recent case study in IC 2574).

Similar structures have been known to exist in spiral galaxies for some time now. Some of them have been the subject of detailed studies, starting with those found in our own Galaxy (Heiles 1979, 1984, 1990) and followed by studies of M31 (Brinks \& Bajaja 1986), M33 (Deul \& den Hartog 1990), M101, and NGC 6946 (Kamphuis 1993). However, in these galaxies it is far from trivial to derive the physical properties of the observed shells. In our Galaxy, the determination of the characteristics of $\mathrm{H}$ I shells is hampered by the difficulty of assigning distances to the shells. Looking at nearby spiral galaxies, however, does not facilitate things too much either, since the structure of their ISM is strongly modified by spiral density waves and differential rotation, both effects dramatically influencing the shape and formation of holes and shells.

Dwarf galaxies, on the other hand, are slow rotators, generally show almost solid-body rotation, and lack density waves. This implies that once features like shells have formed, they are not deformed by galactic shear and therefore tend to be long lived. Moreover, the overall gravitational potential of a dwarf is much lower as compared with a normal spiral. This means that the same amount of energy input of a star-forming region has a much more pronounced impact on the overall appearance of the ISM. Dwarf galaxies also tend to have a thicker disk compared with massive spirals (see $\S 5.2$ ), and the $\mathrm{H}$ I volume density of dwarf galaxies is lower, again facilitating the creation of large holes.

The properties of $\mathrm{H}$ I holes in the ISM have been studied in detail in only a few dwarf galaxies thus far. The ISM of the Magellanic Clouds is the subject of papers by StaveleySmith et al. (1997, SMC) and Kim et al. (1998, LMC). In addition to these very nearby galaxies (which, by the way, are strongly interacting with our galaxy), the ISM of only a few other gas-rich dwarf galaxies has been studied in detail (Ho II: Puche et al. 1992; IC 10: Wilcots \& Miller 1998; IC 2574: Walter \& Brinks 1999; NGC 6822: de Blok \& Walter 2000; Ho I: Ott et al. 2001).

In order to allow a comparison with $\mathrm{H}$ I holes found in other galaxies, we compiled a catalog of holes for DDO 47. We conducted a search for $\mathrm{H}$ I holes by plotting channel maps, looking at movies (using KVIEW in the KARMA package), and interactively inspecting position-velocity cuts (using KPVSLICE within KARMA). Both authors independently searched the $\mathrm{H}$ I cube so as to minimize personal bias. Having said that, until an automated procedure becomes generally available, along the lines of the approach developed by Thilker, Braun, \& Walterbos (1998; see also Mashchenko, Thilker, \& Braun 1999), there will always remain some measure of subjectivity in defining $\mathrm{H} \mathrm{I}$.

The holes in DDO 47 were not as clearly defined as in some of the other dwarf irregular galaxies at similar distances (such as Ho II or IC 2574). This can be attributed to the lower sensitivity of our observations (less observing time, as well as a somewhat larger distance). The larger distance also implies that we cannot detect holes smaller than $\approx 200-250 \mathrm{pc}$ (corresponding to twice the beam size). Because of the small inclination, the holes are most easily recognized in the channel maps, as illustrated in Figures $1 a$ and $1 b$. After careful inspection, we felt confident with the detection of 19 holes (see the discussion below) within the neutral ISM of DDO 47. The expansion velocities were measured using spectra centered at each hole.

Figure 13 shows a plot of the distribution of the $19 \mathrm{H} \mathrm{I}$ holes detected with confidence in DDO 47 (with sequence numbers). The gray-scale map is again a linear representation of the total H I surface brightness. Note that most of the holes show up in the integrated $\mathrm{H}$ I map, whereas some can only be detected in the channel maps or in positionvelocity diagrams.

Table 4 represents the list of the detected $\mathrm{H}$ I holes and their derived properties. Column (1): number of the hole (sorted by right ascension). Columns (2) and (3): position of the center of the hole in right ascension and declination, respectively (B1950.0 coordinates). Typical errors are 3". Columns (4)-(6): the major and minor axis of the holes $\left(b_{\text {maj }}\right.$ and $b_{\min }$, in parsecs), as well as the position angle (in degrees). The accuracy of the size of the holes is around $100 \mathrm{pc}$ (the FWHM of the synthesized beam). Column (7): the diameter of the holes defined as the geometric mean, $d=$ $\left(b_{\text {maj }} b_{\text {min }}\right)^{1 / 2}$. Column (8): heliocentric velocity, $v_{\text {hel }}$, of the hole defined by the channel in which the hole is largest or most prominent. The accuracy of $v_{\text {hel }}$ is about $2.5 \mathrm{~km} \mathrm{~s}^{-1}$ (the velocity resolution of our observations). Column (9): the hole type (1-3), as defined in Brinks \& Bajaja (1986). Column (10): the expansion velocity, $v_{\mathrm{exp}}$, of the hole given in kilometers per second. Note that in the case of a type 1 hole, no expansion velocity can be measured. Typical errors are about $2.5 \mathrm{~km} \mathrm{~s}^{-1}$ (the velocity resolution). Column (11): kinematic age of the hole in units of $10^{6} \mathrm{yr}$. This age determination assumes that over its entire lifetime, the hole was expanding with $v_{\text {exp }}$; hence, $t=d /\left(2 v_{\text {exp }}\right)$. The kinetic age is therefore an upper limit for the actual age of the hole. The accuracy of the kinetic age is typically $20 \%$. Column (12): indicative $\mathrm{H}$ I mass $m_{\mathrm{H}}$ in units of $10^{4} M_{\odot}$. It is the mass that filled the observed hole before the evacuation (assuming that the hole is completely evacuated now). As the volume, we took $V=(4 / 3) \pi(1 / 2) b_{\text {maj }}(1 / 2) b_{\text {min }}(1 / 2) d$ 
TABLE 4

Catalog of H i Holes in DDO 47

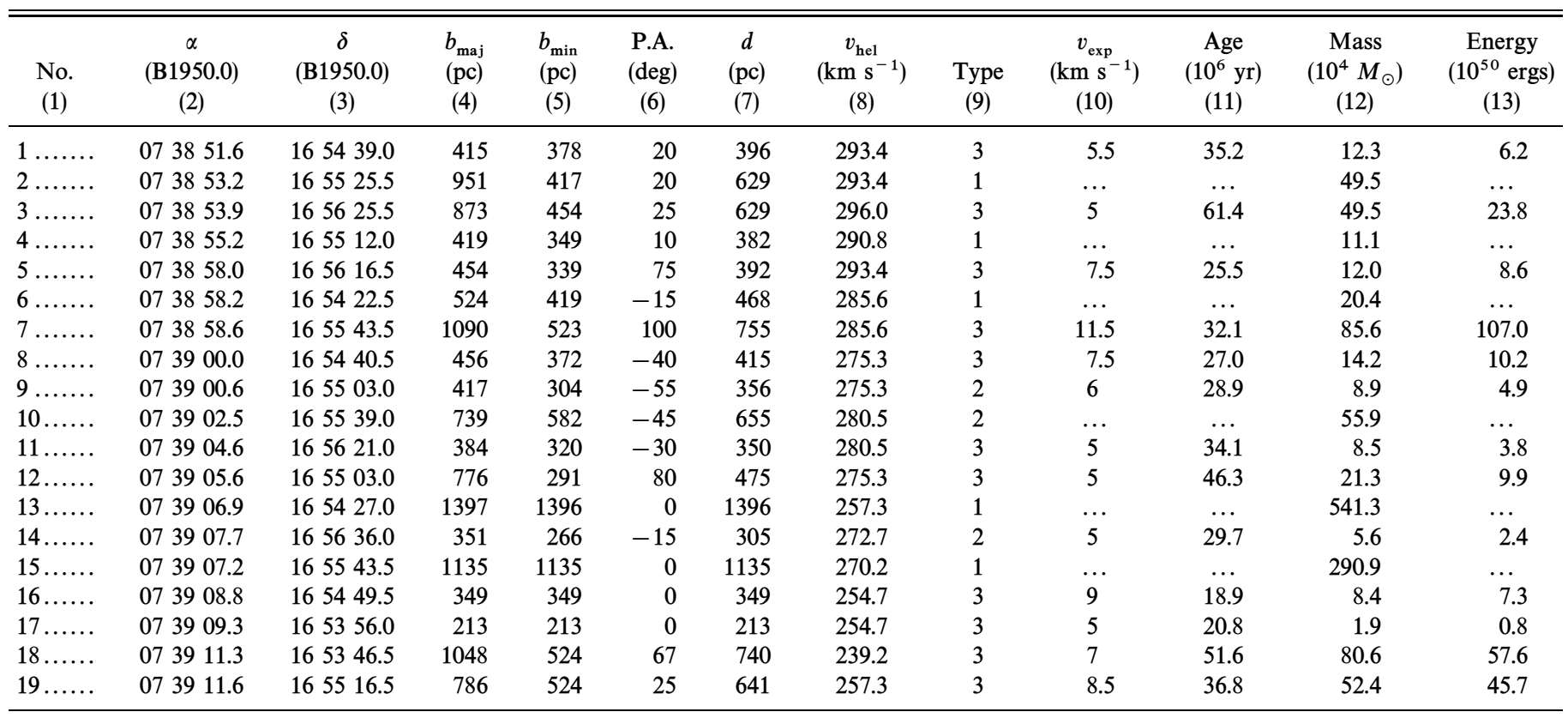

NoTE.- -Units of right ascension are hours, minutes, and seconds, and units of declination are degrees, arcminutes, and arcseconds.

(where $d$ is defined in col. [7]). An average $\mathrm{H}$ I volume density was adopted, taking the average surface density of $3.5 M_{\odot} \mathrm{pc}^{-2}(\S 4.1)$ and dividing it by $2 \pi^{1 / 2}$ times the $1 \sigma$ scale height of the H I gas $(\sim 500 \mathrm{pc}$; see $\S 5.2)$. This results in an average $\mathrm{H}$ I volume density of $\sim 0.15 \mathrm{~cm}^{-3}$. To correct this value for the amount of primordial helium, a factor of 1.35 has to be applied. The relative error is of the order of $30 \%$. Column (13): the energy that was needed to produce a hole in units of $10^{50}$ ergs. We used the equation first given by Heiles (1979) to calculate this number,

$$
E_{E}=5.3 \times 10^{43} n_{0}^{1.12}(d / 2)^{3.12} v_{\mathrm{exp}}^{1.4} \mathrm{ergs},
$$

where $v_{\text {exp }}$ is the expansion velocity in kilometers per second, $n_{0}$ is the density of the ambient medium in particles per cubic centimeter $\left(n_{0}=0.15 \mathrm{~cm}^{-3}\right)$, and $d$ is the diameter of the shell in parsecs. Note that the above equation is often referred to as Chevalier's equation. The relative error of $E_{E}$ is rather large and is of the order of $50 \%$ (for a discussion, see Walter \& Brinks 1999).

We should like to note that the diameters, expansion velocities, and energies needed to create the holes agree well with the $\mathrm{H}$ I hole properties found in other irregular galaxies (Walter \& Brinks 1999). This reinforces the notion that, at least to first order, the energy deposited by an OB association in the form of $\mathrm{SN}$ explosions is independent of the type of the host galaxy; the range of energies supposedly necessary to create the holes being the same for a dwarf galaxy, as well as for a large, spiral galaxy. Holes in dwarf galaxies are larger by a factor of a few as compared with those in disk galaxies because of a number of factors that all work in the same direction. First of all, the gravitational potential in a dwarf galaxy is smaller. As a consequence of that, the $\mathrm{H}$ I scale height is larger and the volume density lower. So, for the same energy input, an $\mathrm{H}$ I shell can grow to larger dimensions. As dwarfs lack spiral density waves and differential rotation over much of their body, the shells can persist for longer times.

\section{H I-H $\alpha$ CONNECTION OF DDO 47}

The theory of stochastic self-propagating star formation predicts that new sites of star formation form on the rims of expanding shells. The idea behind this is that the ambient material is swept up due to the expansion of a supernovadriven shell until the mass density on the rim becomes large enough that gravitational fragmentation can lead to the onset of secondary star formation. To check the validity of this picture, one therefore has to compare the sites of current star formation with the positions and sizes of the $\mathrm{H} \mathrm{I}$ holes to see whether there exists such a correlation or not. Since $\mathrm{H} \alpha$ emission is a good tracer for star formation in a galaxy and the $\mathrm{H}$ i holes are believed to be the aftermath of a previous star formation episode, it therefore makes sense to compare the positions of $\mathrm{H}$ II regions with the $\mathrm{H}$ I holes detected by us within DDO 47. As mentioned before, we will rely on the results of Strobel et al. (1991), who cataloged the brightest $\mathrm{H}$ II regions of DDO 47. Figure 14 shows an overlay of their $\mathrm{H}$ II regions (filled squares) with the $\mathrm{H}$ I surface density map of DDO 47 . The contours are plotted at column densities of $1,5,9$, and $14 \times 10^{20} \mathrm{~cm}^{-2}$. Since we are only interested in the positions of the $\mathrm{H}$ II regions, the sizes of the boxes merely indicate the location, without attempting to represent the flux of the individual $\mathrm{H}$ II regions. A comparison of this figure with the position of the holes (Fig. 13) shows that the situation found in DDO 47 qualitatively fits the picture described above of propagating star formation. Most of the $\mathrm{H}$ II regions that are detected in DDO 47 fall on the rims of the $\mathrm{H}$ I holes. The $\mathrm{H}$ I surface density in the immediate surrounding of the sites of active star formation is, in almost all cases, higher than $8 \times 10^{20}$ $\mathrm{cm}^{-2}$, similar to what has been found empirically (see, e.g., Skillman 1987; Kennicutt 1989). It should be noted that this empirical threshold is a necessary condition for star formation to commence but not a sufficient one. There are some locations where the $\mathrm{H}$ I has reached surface densities as high as the threshold or even higher without any associated star 
formation being evident. Either star formation is just about to commence or, more likely, some other condition, such as temperature of the gas or velocity dispersion, is preventing the onset of a burst.

\section{SUMMARY AND CONCLUSIONS}

We have presented a detailed $\mathrm{H}$ I study of the dwarf galaxy DDO 47 (UGC 3974). The main results of this investigation can be summarized as follows:

1. DDO 47 is a gas-rich dwarf irregular galaxy. Adopting a distance of $4 \mathrm{Mpc}$, we derive an $\mathrm{H}$ I mass of $2.3 \times 10^{8} \mathrm{M}_{\odot}$, within $10 \%$ of the value based on the Arecibo measurements published by Hoffman et al. (1996). Its apparent $B$ magnitude is $13.5 \mathrm{mag}$, which corresponds to an absolute blue magnitude of $-14.5 \mathrm{mag}$. DDO 47 therefore falls well within the definition of a dwarf galaxy. The $\mathrm{H}$ I mass-toblue light ratio of DDO 47 is high at $M_{\mathrm{H}} / L_{B}=2.4$ $M_{\odot} / L_{B \odot}$.

2. Mapping of a large field around DDO 47 resulted in the detection of a companion galaxy at almost the same systemic velocity. In our search for an optical counterpart, we found a faint galaxy at the location where $\mathrm{H}$ I was detected; this object had been cataloged previously as CGCG 087-033. Its $\mathrm{H}$ I mass is only about $6 \%$ of that of the parent galaxy $\left(1.4 \times 10^{7} M_{\odot}\right)$, and its blue luminosity is $1.3 \times 10^{7} L_{B \odot}$, leading to an $\mathrm{H}$ I mass-to-blue light ratio of $M_{\mathrm{H}} / L_{B}=1.1 M_{\odot} / L_{B \odot}$. The inclination of this object being unknown, we estimate a lower limit to the dynamical mass of $M_{\mathrm{dyn}}>1.2 \times 10^{8} M_{\odot}$. The very similar redshift suggests that both galaxies are in the plane of the sky and that their linear separation is $\approx 20 \mathrm{kpc}$.

3. An analysis of the rotation curve of DDO 47 shows that the observed mass in the form of stars and gas is not able to explain the overall dynamics. In fact, about $80 \%$ of the dynamical mass of DDO 47 seems to be in the form of dark matter, $M_{\mathrm{dyn}}=5.1 \times 10^{9} M_{\odot}$.
4. Using a simple mass model for DDO 47, we derive the thickness of its $\mathrm{H}$ I disk. The result is a $1 \sigma$ scale height of about $500 \mathrm{pc}$, which is larger than that compared with the values typically found in spiral galaxies but similar to the thicknesses derived for other dwarf galaxies, such as NGC 6822, Ho I, Ho II, or IC 2574.

5. In total, $19 \mathrm{H}$ I holes were detected with confidence. Only in $50 \%$ of all cases were we able to measure an expansion velocity. The sizes, expansion velocities and energies needed to create the holes are similar to the $\mathrm{H}$ I hole properties found in other gas-rich dwarf galaxies (Ho II and IC 2574).

6. Most of the $\mathrm{H}$ II regions within DDO 47 are found near the rims of the $\mathrm{H}$ I shells, where column densities exceed values of $8 \times 10^{20} \mathrm{~cm}^{-2}$. The threshold seems to represent a necessary but not sufficient condition for star formation to commence.

In summary, the neutral interstellar medium of DDO 47 exhibits the typical structure seen in other dwarf irregular galaxies studied at similarly high resolution. Although today's SF rate is modest, a likely recent interaction with the nearby companion may have triggered a burst of SF in the past, which may have created the prominent $\mathrm{H}$ I holes and shells that are visible in DDO 47's ISM today.

F. W. acknowledges NSF grant AST 96-13717 and EB grants awarded by CONACYT (grants 0460P-E and 27606-E). We thank Jürgen Ott for help with the optical data reduction. This research has made use of the NASA/ IPAC Extragalactic Database (NED), which is operated by the Jet Propulsion Laboratory, California Institute of Technology, under contract with the National Aeronautics and Space Administration (NASA). The authors also acknowledge the use of NASA's Astrophysical Data System Abstract Service (ADS) and NASA's SkyView.

\section{REFERENCES}

Baars, J. W. M., Genzel, R., Pauliny-Toth, I. I. K., \& Witzel, A. 1977, A\&A, 61,99

Begeman, K. G. 1989, A\&A, 223, 47

Briggs, D. 1995, Ph.D. thesis, New Mexico Inst. Mining Technol.

Brinks, E. 1981, A\&A, 95, L1

Brinks, E., \& Bajaja, E. 1986, A\&A, 169, 14

Brinks, E., \& Burton, W. B. 1984, A\&A, 141, 195

Brinks, E., \& Walter, F. 1998, in Proc. Bonn/Bochum-Graduiertenkolleg

Workshop, The Magellanic Clouds and Other Dwarf Galaxies, ed.

T. Richtler \& J. M. Braun (Bonn: Shaker), 1

Broeils, A. 1992, Ph.D. thesis, Univ. Groningen

Campos-Aguilar, A., Moles, M., \& Masegose, J. 1993, AJ, 106, 1784

Clark, B. G. 1980, A\&A, 89, 377

de Blok, W. J. G., \& Walter, F. 2000, ApJ, 537, L95

Deul, E. R., \& den Hartog, R. H. 1990, A\&A, 229, 362

de Vaucouleurs, G., de Vaucouleurs, A., \& Buta, R. J. 1983, AJ, 88, 764

de Vaucouleurs, G., de Vaucouleurs, A., Corwin, H. G., Jr., Buta, R. J.,

Paturel, G., \& Fouqué, P. 1991, Third Reference Catalogue of Bright Galaxies (New York: Springer)

Georgiev, Ts. B., Karachentsev, I. D., \& Tikhonov, N. A. 1997, Astron. Lett., 23, 514

Gerola, H., \& Seiden, P. E. 1978, ApJ, 223, 129

Heiles, C. 1979, ApJ, 229, 533 1984, ApJS, 55, 585 .1990, ApJ, 354, 483

Hoffman, G. L., Salpeter, E. E., Farhat, B., Roos, T., Williams, H., \& Helou, G. 1996, ApJS, 105, 269

Högbom, J. A. 1974, A\&AS, 15, 417

Huchtmeier, W. K., \& Richter, O.-G. 1988, A\&A, 203, 237

Hunter, D. A., \& Gallagher, J. S., III. 1985a, AJ, 90, 1789 1985 b, ApJS, 58, 533

Hunter, D. A., Hawley, W. N., \& Gallagher, J. S., III. 1993, AJ, 106, 1797

Jörsäter, S., \& van Moorsel, G. A. 1995, AJ, 110, 2037

Kamphuis, J. J. 1993, Ph.D. thesis, Univ. Groningen
Karachentseva, V. E., \& Karachentsev, I. D. 1998, A\&AS, 127, 409

Kennicutt, R. C. 1989, ApJ, 344, 685

Kim, S., Staveley-Smith, L., Dopita, M. A., Freeman, K. C., Sault, R. J., Kesteven, M. J., \& McConnell, D. 1998, ApJ, 503, 674

Kraan-Korteweg, R. 1986, A\&AS, 66, 255

Lewis, B. M., Helou, G., \& Salpeter, E. E. 1985, ApJS, 59, 161

Mashchenko, S. Y., Thilker, D. A., \& Braun, R. 1999, A\&A, 343, 352

McGaugh, S. S., \& de Blok, W. J. G. 1997, ApJ, 481, 689

Mueller, M. W., \& Arnett, W. D. 1976, ApJ, 210, 670

Ott, J., Walter, F., Brinks, E., van Dyk, S., Dirsch, B., \& Klein, U. 2001, AJ, submitted

Palouš, J. 1998, in Proc. Bonn/Bochum-Graduiertenkolleg Workshop, The Magellanic Clouds and Other Dwarf Galaxies, ed. T. Richtler \& J. Braun (Bonn: Shaker), 67

Puche, D., Westpfahl, D., Brinks, E., \& Roy, J.-R. 1992, AJ, 103, 1841

Rhode, K. L., Salzer, J. J., \& Westpfahl, D. J. 1999, AJ, 118, 323

Skillman, E. D. 1987, in Star Formation in Galaxies, ed. C. J. Lonsdale Persson (NASA CP-2466) (Washington: NASA), 263

Skillman, E. D., Kennicutt, R. C., \& Hodge, P. W. 1989, ApJ, 347, 875

Staveley-Smith, L., Davies, R. D., \& Kinman, T. D. 1992, MNRAS, 258, 334

Staveley-Smith, L., Sault, K. J. Hatzidimitriou, D., Kesteven, M. J., \& McConnell, D. 1997, MNRAS, 289, 225

Stewart, S. G., \& Walter, F. 2000, AJ, 120, 1794

Stil, J. M. 1999, Ph.D. thesis, Univ. Leiden

Strobel, N. V., Hodge, P., \& Kennicutt, R. C., Jr. 1991, ApJ, 383, 148

Swaters, R. A., Sancisi, R., van Albada, T. S., \& van der Hulst, J. M. 1998, BAAS, 193, 2106

Tacconi, L. J., \& Young, J. S. 1987, ApJ, 322, 681

Taylor, C. 1997, ApJ, 480, 524

Telles, E., \& Maddox, S. J. 2000, MNRAS, 311, 307

Telles, E., \& Terlevich, R. 1995, MNRAS, 275, 1

Tenorio-Tagle, G., \& Bodenheimer, P. 1988, ARA\&A, 26, 145

Thilker, D. A., Braun, R., \& Walterbos, R. M. 1998, A\&A, 332, 429 
van der Hulst, J. M. 1996, in ASP Conf. Ser. 106, The Minnesota Lectures on Extragalactic Neutral Hydrogen, ed. E. D. Skillman (San Francisco: ASP), 47

Walter, F., \& Brinks, E. 1999, AJ, 118, 273
Walter, F., Brinks, E., Duric, N., \& Klein, U. 1997, AJ, 113, 2031

Wilcots, E. M., \& Miller, B. W. 1998, AJ, 116, 2363

Young, J. S., et al. 1995, ApJS, 98, 219

Youngblood, A. J., \& Hunter, D. A. 1999, ApJ, 519, 55 